\title{
Definitions of Predictability for Cyber Physical Systems
}

\author{
Beilei Sun ${ }^{1}$, Xi Li ${ }^{1, *}$, Bo Wan, Chao Wang, Xuehai Zhou, Xianglan Chen \\ University of Science and Technology of China, Hefei, China
}

\begin{abstract}
With the recent proliferation of different types of Cyber Physical Systems (CPS), it is critically important to investigate the predictability of such systems. Along with functional correctness of the components, these systems must also ensure that timing and delay constraints of components are properly for the entire system to behave in a predictable manner in presence of various kinds of uncertainties. While the functional correctness of the CPS components has been investigated in the past, very little is available about the timing issues. The objective of this paper is to conduct an investigation of key issues involved to ensure the predictability of the system, introduce rigorous definitions of performance parameters, and propose metrics for their evaluation and analyze their suitability to be used in the presence of uncertainties in which CPS operate. The results are expected to provide greater insight into the time critical behavior of CPS components.
\end{abstract}

Keywords: Cyber Physical Systems, Predictability, I/O Behavior Predictability, Time Predictability, Order Predictability

\section{Introduction}

Cyber-Physical Systems (CPS) are integrations of computation with physical processes. Embedded computers and networks monitor and control the physical processes, with feedback loops where physical processes affect computations and vice versa [38, 40]. Predictability is crucial for CPS testing and

${ }^{*}$ Corresponding Author. Address: School of Computer Science, University of Science and Technology of China, Room 421, Electronic Building 3rd, West Campus USTC, Hefei 215123, China. Tel: +86051262888062.

Email address: 1lxx@.ustc.edu.cn (Xi Li ) 
verification, which are required by time-critical applications such as traffic control, automotive safety, and health care systems [20, 38, 40, 41, 45]. To improve the predictability of concurrent and preemptive CPS, the first and foremost work is to figure out the properties that should be predictable in CPS and precisely define the concept of predictability.

The predictability problem resounds throughout the embedded systems community, particularly throughout the real-time community. Stankovic and Ramamritham discuss the need for predictability, with respect to the time requirements of different kinds of systems [52]. Thiele and Wilhelm define the time predictability by considering the difference between the real WorstCase-Execution-Time (WCET) and the WCET bound, which is often called the pessimism of the analysis, as a measure for predictability [53]. Kirner and Puschner define predictability as the interval between the Best Case Execution Time (BCET) and the WCET, where a smaller interval means better predictability [28]. Grund et al. investigate and propose a template for the predictability definitions, and define the predictability as the quotient of the minimum execution time over the maximum execution time with all the possible inputs [21]. The state-of-the-art predictability definitions can be classified into four categories: 1) interval between BCET and WCET [28]; 2) quotient of BCET over WCET [21]; 3) quotient of WCET over WCET bound [53]; 4) quotient of BCET over WCET bound [53]. Almost all the aforementioned definitions of predictability are based on WCET and BCET, which are estimated by assuming the uninterrupted execution of a single task [4, 21, 28, 53, 56]. However, the assumption is not true for most of CPS due to the concurrent and preemptive nature of CPS [38]. In addition, CPS must be robust in presence of unexpected conditions, and adaptable to subsystem failures. Therefore, the predictability of CPS has more rigorous semantic requirements than the above definitions [40].

The objective of this paper is to investigate issues involved to ensure the time predictability of CPS, introduce rigorous definitions of performance parameters, and propose metrics for their evaluation and analyze their suitability to be used in the presence of uncertainties in which Cyber Physical Systems operate. Through analyzing the behaviors of I/O and function task of typical programming models and characteristics of CPS, we argue that both the function tasks and I/O must have time predictability and order predictability. The I/O behaviors must be predictable in terms of their ready time and the Inputs ready order, where the ready time refers to the interval between the time when the value in the $\mathrm{I} / \mathrm{O}$ source changes and the time 
when the value in the I/O port is modified correspondingly. The predictability of function tasks are discussed and defined according to the concurrent and preemptive characteristics of CPS. It includes the time predictability of individual task and tasks set. And the execution order predictability of tasks in a scheduling set must be predictable as well. We investigate the sources of uncertainties of different properties that must be predictable, and propose definitions for them based on the uncertainties and the template introduced in [21. Table-1] shows the differences between the existing definitions and our definitions. The major contributions of our work are as follows:

(1) We give a comprehensive catalog of possible unpredictability and summarize properties that must be predictable. We emphasize the importance of I/O predictability by analyzing their behaviors in different programming models, and we analyze the function task predictability requirements in the concurrent and preemptive environments.

(2) A careful investigation of the uncertainty sources of different properties is made. By analyzing the major uncertainties, we propose metrics and formal predictability definitions for those properties. We present methods to calculate parameters that are used in the definitions.

(3) To the best of our knowledge, this paper is the first to stress the importance of order predictability in addition to only time predictability reported in the literature. We propose new ideas to determine the order (i.e., the inputs ready order and the tasks execution order), and present methods to calculate their predictability.

Our definitions can serve as metrics for measuring and comparing the predictability of CPS. CPS that have greater predictability tend to have more reliable testing and verification results, as well as time safety. When applied during CPS design, the metrics help the designer in selecting suitable programming models, runtime systems and hardware platforms to satisfy safety as well as energy efficiency requirements. The order predictability can also help to improve the CPS design, i.e., a predictable inputs ready order is critical for the predictability of the input-determined real-time systems, and the task execution order is important to make the scheduling algorithm predictable and avoid some scheduling anomalies and the mutex hazards as well. Finally, since our predictability definitions capture major sources of uncertainties, they can be used to find out the bottleneck of the predictability. The designer can improve the system's predictability effectively by optimizing the bottleneck. 
Table 1: A comparison between the existing predictability definitions and our definitions

\begin{tabular}{|c|c|c|c|c|c|}
\hline & Existing & \multicolumn{4}{|c|}{ Our Definitions } \\
\hline Behaviors & $\begin{array}{c}\text { Function } \\
\text { task }\end{array}$ & \multicolumn{2}{|c|}{ Function task } & \multicolumn{2}{|c|}{$\mathrm{I} / \mathrm{O}$} \\
\hline Properties & $\begin{array}{l}\text { Execution } \\
\text { time }\end{array}$ & $\begin{array}{c}\text { Response } \\
\text { time }\end{array}$ & $\begin{array}{c}\text { Execution } \\
\text { Order }\end{array}$ & $\begin{array}{c}\text { I/O Ready } \\
\text { time }\end{array}$ & $\begin{array}{c}\text { Inputs } \\
\text { ready order }\end{array}$ \\
\hline $\begin{array}{l}\text { Sources of } \\
\text { Uncer- } \\
\text { tainties }\end{array}$ & $\begin{array}{c}\text { Inputs, } \\
\text { Hardware } \\
\text { states }\end{array}$ & $\begin{array}{l}\text { Inputs, Ha } \\
\text { Concurrenc } \\
\text { Scheduling, } \\
\text { tem, Mutex }\end{array}$ & $\begin{array}{l}\text { vare states, } \\
\text { Preemption, } \\
\text { untime sys- } \\
\text { zards }\end{array}$ & $\begin{array}{c}\text { Sensor, } \\
\text { Network, } \\
\text { Memory } \\
\text { delay }\end{array}$ & $\begin{array}{c}\text { Inputs } \\
\text { ready time, } \\
\text { processing } \\
\text { order }\end{array}$ \\
\hline Metrics & $\begin{array}{c}\text { Task } \\
\text { execution } \\
\text { time } \\
\text { variability }\end{array}$ & $\begin{array}{c}\text { Task } \\
\text { response } \\
\text { time } \\
\text { variability }\end{array}$ & $\begin{array}{c}\text { Execution } \\
\text { order } \\
\text { distance } \\
\text { variability }\end{array}$ & $\begin{array}{c}\text { Inputs } \\
\text { ready time } \\
\text { variability }\end{array}$ & $\begin{array}{c}\text { Inputs } \\
\text { buffer costs } \\
\text { variability }\end{array}$ \\
\hline
\end{tabular}

The rest of this paper is organized as follows: Section 2 discusses the requirements of the predictability of CPS and summarizes the behaviors and properties that must to be predictable. We investigate the sources of uncertainties of each predictable property, and present formal definitions in Section 3. Section 4 shows the related works, and we conclude our work and show the future work in Section 5 .

\section{Predictability Requirements of CPS}

In this section, we illustrate that both the function task behaviors and the I/O behaviors must be predictable by analyzing their logical and real time performance in different real-time programming models at first. Then, from the characteristics of CPS standpoint, we argue that both the time property and the order property of the different behaviors must be predictable.

According to Klein and Ralya [31], Input is defined as reading data from one or more sources of input; Output is defined as writing the results of the computation to one or more sinks, which may be devices and/or main memory; function task is defined as the process to compute output values, which are functions of the gathered input values. These definitions are used throughout this paper. 


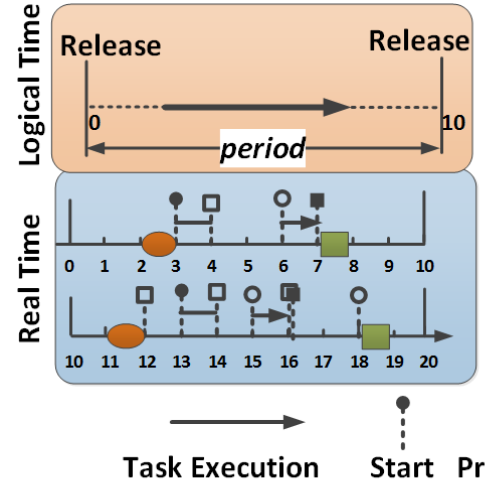

(a) Asynchronous Model

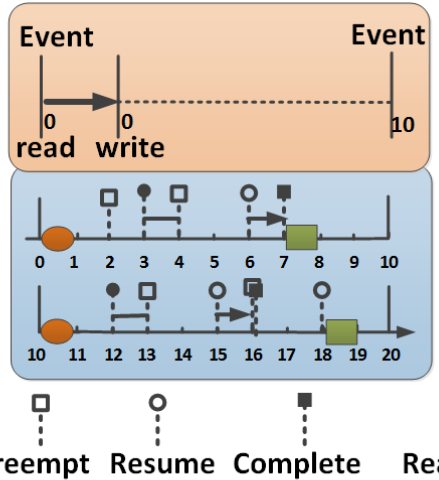

(b) Synchronous Model

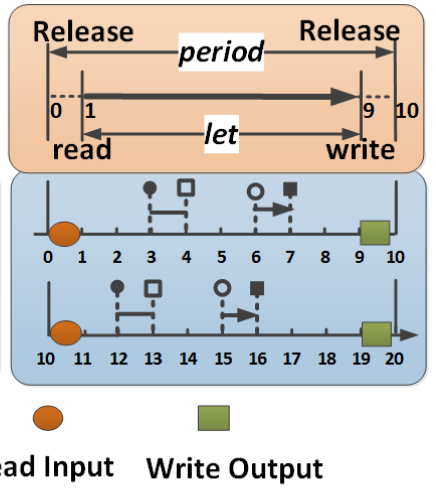

(c) Timed Model

Figure 1: Logical abstraction and real time execution of different programming models

\subsection{Behaviors Need to be Predictable}

Most of the real-time programs are input-determined programs, i.e., if, for all sequences of input values and times, the program produces, in all runs, unique sequences of output values and times [29]. Therefore the I/O behaviors are critical to improve the predictability of the system. However, the I/O behaviors of most of the real-time programs are unpredictable in either the logical time (specified by programming models) or the real time (performed during run time). We use the example shown in Fig.1 to analyze the I/O behaviors of a task, which is realized in different programming models and executed in preemptive environments. The top half of Fig.1 shows the logical time of three real-time programming models (summarized in [16]). The bottom half shows the execution of a task in different real time periods, during which the task can be preempted by other tasks.

The asynchronous model bounds execution times using deadlines. A system built on the asynchronous model is a set of programs that consist of a finite number of tasks, which execute concurrently under the supervision of specific mechanisms such as real-time operating systems [16]. The execution of asynchronous program is correct if the tasks finish writing outputs before deadline. However, since no I/O behaviors are specified in the asynchronous model, the I/O behaviors are highly unpredictable in both the logical time and the real time. As shown in Fig.1 (a), the time to process I/O is not specified in the programming model. In the first real time period, the input and output happen at the $2^{\text {nd }}$ time instant and the $7^{\text {th }}$ time instant, respectively, 
while in the second real time period, they are processed at the $1^{\text {st }}$ time instant and the $8^{\text {th }}$ time instant, respectively. In the synchronous model, the logical temporal reference is completely determined by the successive reactions of the system happening on the occurrences of observed events [8, 23]. Due to the synchronous assumption of the model, the I/O behavior is predictable in the logical time. In the real time, the input is processed at fixed time instant, such as PRET-C [2]. However, since the synchronous program is correct as long as the output completes before the next input, the output behavior is still not predictable in the real time. As shown in Fig.1. (b), the logical time to read input is specified in the programming model. Therefore, the input is read at beginning of each real time period. However, the output is written at the $7^{\text {th }}$ time instant and the $8^{\text {th }}$ time instant in different real time periods, respectively. The timed model (e.g., the Logical Execution Model (LET) [30]) describes the system with logical duration information, which is specified by the designer [16, 30]. Since the time information is priori fixed, e.g., the times to read input and to write output are fixed in the LET model, the I/O behaviors of the LET model are predictable and composable both in the real time and the logical time [29, 30]. As shown in Fig.1 (c), the logical times to read input and write output are specified in the programming model. In different real time periods, the input is read at the beginning and the output is written at the last time instant.

Both of the logical and real time I/O behaviors have significant influences on the behaviors of the real-time system. Therefore, the predictability must not be limited to the function tasks, the I/O behaviors must be predictable as well. Though the predictability of function tasks have been researched extensively, the predictability of the I/O behaviors of a task is not well studied.

\subsection{Predictable Properties of Different Behaviors}

In this section, from the standpoint of emerging characteristics of CPS, we argue that both the order property and the time property of different behaviors must be predictable.

\subsubsection{Predictable Properties of $I / O$ behaviors}

CPS have diverse kinds of I/Os, which must be processed timely and safely. Meanwhile, a complex CPS is always composed by different sub-systems, which are connected through I/Os. Therefore, more rigorous properties of I/O behaviors need to be predictable. We argue that the input ready time, the inputs ready order, and the time of writing output must be predictable. 


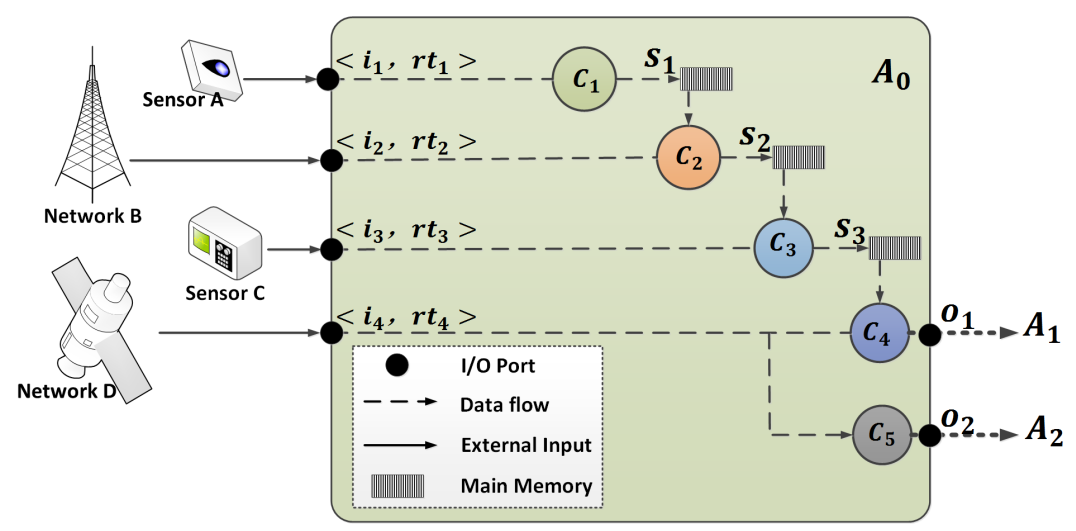

Figure 2: Illustration of input-ready order and input-ready time

To be concise, we take Fig, 2 for example. $A_{0} \sim A_{2}$ are underlying implementations of actors, which are concurrent components that communicate through ports and interact according to a common pattern of interaction [43]. $i_{1} \sim i_{4}$ are the input ports of $A_{0}$. The sources of inputs vary from highly sensitive sensor to satellite network. The ready time of input $i$ is the interval between the time when the value in the sensor (Sensor $A$ or $C$ ) or network (Network $B$ or $D$ ) changes and the time when the value of the input port $i$ is modified correspondingly and ready for the use of the function task. $<i_{j}, r t_{j}>$ represents that the time input $i_{j}$ becomes ready must be earlier than time $r t_{j} . C_{1} \sim C_{5}$ are function tasks, whose inputs include the external inputs $\left(i_{1}, i_{2}, i_{3}\right.$, and $\left.i_{4}\right)$ and the signals $\left(s_{1}, s_{2}\right.$, and $\left.s_{3}\right)$ which are written back to the main memory by its precedent tasks. The input data must be buffered in the input port unless the time an input become ready equals to the time the value in the input port is read. $s_{1} \sim s_{3}$ are the signals emitted by function tasks. They are called as local outputs, because their values are only updated in the main memory and used by other tasks in the same actor. $o_{1}$ and $o_{2}$ are output ports of actor $A_{0}$. Since their values will be broadcasted to other actors through the network, they are called as the network outputs.

The input ready time must be predictable. The time an input may vary greatly, because the preparation of the input may be affected by other events, such as network transmission jitter and the sensor response time jitter. An overdue input leads to the delay of the computing of the function tasks, which in turn leads to the deadline missing of the function task. For example, in Fig.2, if $i_{1}$ cannot become ready before $r t_{1}$, task $C_{1}$ may miss its deadline. 
The inputs ready order must be predictable. The first reason is because different input ready orders result in different system behaviors. The evolution of the real-time programming models shows that the I/O behaviors play a more and more important role [29]. The asynchronous model does not specify the I/O behaviors. Even though the inputs processing sequences are specified in the programs built on the synchronous model and timed model, the inputs may become ready in an unexpected order, which is different from the specification. For example, in Fig,2, to get the value of $o_{1}$, the inputs reading sequence specified in the program is $i_{1} \rightarrow i_{2} \rightarrow i_{3} \rightarrow i_{4}$, where $i_{1} \rightarrow i_{2}$ means that $i_{1}$ is processed earlier than $i_{2}$. If the inputs become ready in the same order as the specification, $A_{0}$ writes $o_{1}$ first, and $o_{2}$ next. $o_{1}$ triggers the events of $A_{1}$, and $o_{2}$ triggers the events of $A_{2}$. However, if the inputs become ready in the $i_{1} \rightarrow i_{3} \rightarrow i_{4} \rightarrow i_{2}$ order, $A_{0}$ can write $o_{2}$ when $i_{4}$ is ready, but cannot write $o_{1}$ until $i_{2}$ become ready in the end. In this case, events of $A_{2}$ are triggered at first. Since $A_{1}$ and $A_{2}$ will create different chain reactions, the different triggering orders of $A_{1}$ and $A_{2}$ will significantly affect the behaviors of the system.

The second reason of the needs for inputs ready order predictability is because of the uncertainties caused by the buffer management costs for the out-of-order inputs. In Fig,2, if the function task can process the input value immediately when the input becomes ready, no buffer is needed. Otherwise, a buffer is needed to store the value of the input until it is processed. For example, if the ready time of $i_{4}$ is earlier than those of $i_{1}, i_{2}$, or $i_{3}$, then the value of $i_{4}$ has to be buffered, and waits for the other inputs to become ready. Experiments have shown that the temporal variability caused by the unpredictable runtime system activities is more serious than the datadependency of the task [54. Since the buffer manager is one of the important runtime system activities [33, the unpredictable inputs ready orders decrease the predictability of the runtime system. This situation is even worse in CPS, which receive diverse kinds of inputs from the physical world.

The time of writing outputs must be predictable. The output values cannot be used until they are written back to main memory (such as values of $s_{1}, s_{2}$, and $s_{3}$ ) or broadcasted to other actors (such as values of $o_{1}$ and $o_{2}$ ). Since output is also a source of input, the predictability of outputs directly influence the predictability of inputs. However, the time of writing outputs is not fixed because of the following two reasons: First, in the synchronous programming model and the asynchronous model, the time to write output is not specified, 


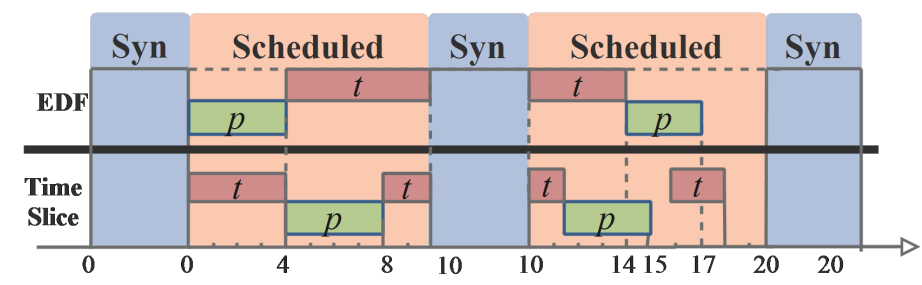

Figure 3: Execution of $p$ and $t$ under different schedulers

and it depends on its function task, which is highly unpredictable (Fig.1 (a) and (b)). Second, even though in the timed model, such as the LET model, the time to write output is pre-determined in the model (Fig.1(c)), the time of writing outputs is still not fixed, because the out writing process can be affected by hardware system, such as the memory system and the networks. Therefore, we need to define and evaluate the time predictability of the outputs.

\subsubsection{Predictable Properties of Function Task Behaviors}

Due to the concurrent and preemptive characteristics of CPS, we argue that the time predictability of function tasks should not be measured by the execution time, which is estimated under the uninterrupted execution assumption [28]. In addition, the execution order of tasks in a scheduling set also needs to be predictable.

The time predictability of function task must not be limited to the predictability of the execution time. For example, in Fig.3, task $t$ and task $p$ are two independent tasks in the same task set, which is executed periodically (10m$\mathrm{s})$. The upper half and lower half of Fig 3 shows the resulting times of task $p$ and task $t$ in different schedulers. Whenever a scheduling event occurs, the Earliest-Deadline-First (EDF) scheduler chooses the task with closest deadline to execute in the next period. The Time-Slice scheduler runs once every time slice to choose the next task to run according to the task priority [12]. We can see that the finish times of task $p$ and task $t$ vary greatly under different schedulers, and even in different periods of the same scheduler. Therefore, in the multi-tasking and preemptive system, e.g., CPS, the definition of the time predictability of function tasks should take the interruptions from other tasks and the overheads of the kernel behaviors into consideration. 


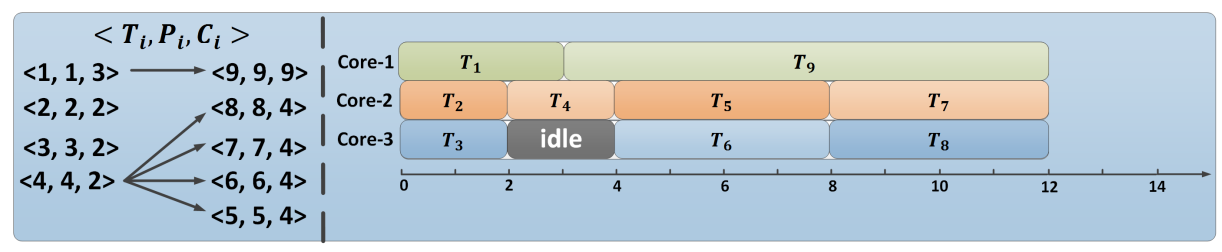

(a) Priority Order: $T_{1}>T_{2}>T_{3}>T_{4}>T_{5}>T_{6}>T_{7}>T_{8}>T_{9}$

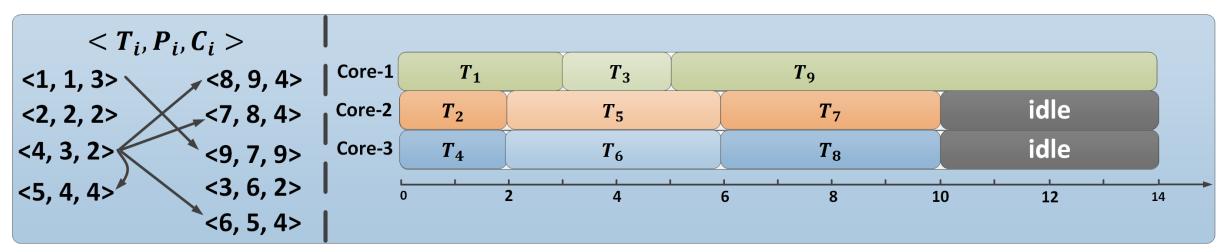

(b) Priority Order: $T_{1}>T_{2}>T_{4}>T_{5}>T_{6}>T_{3}>T_{9}>T_{7}>T_{8}$

Figure 4: Scheduling anomaly while changing priority orders

The execution order of tasks in a scheduling set must be predictable. In the Preemptable, Non-migratable and Fixed priority (P/N/F) cases, the execution of independent tasks is not predictable for arbitrary priority assignments. It is predictable when the tasks are scheduled in the order in which they are released [22]. A non-predictable scheduling algorithm would cause anomalies. Fig 4 and Fig 5 show two of the famous Richard's anomalies [19]. There are 9 tasks with precedence: $T_{9}$ depends on $T_{1} . T_{5}, T_{6}, T_{7}$, and $T_{8}$ depend on $T_{4}$. $<T_{i}, P_{i}, C_{i}>$ represents that the priority of task $T_{i}$ is $P_{i}$, and its computation time is $C_{i}$. The scheduling length of Fig.4(a) is 12 . However, when the execution order changes, the scheduling length increases to 14 (Fig,4(b)). In Fig.5 (a), while the execution time of each task is reduced by 1 and all other parameters remain unchanged, the scheduling length increases. Among all those anomalies, we can observe the change of the execution order. However, in some anomalies, if the execution order is properly arranged, the scheduling length will not increase. For example, the execution order of Fig 5 (b) is the same with that of Fig.4 (a), the scheduling length is 10, which is shorter than the length of Fig 4 (a). Therefore, the execution order is important to make the scheduling algorithms predictable.

Though the execution order is important, it is nondeterministic because of the following reasons: First, if the priority is not assigned according to the release order, the execution order is unpredictable [22]. Second, the "best-effort" scheduler and runtime systems may change the execution order 


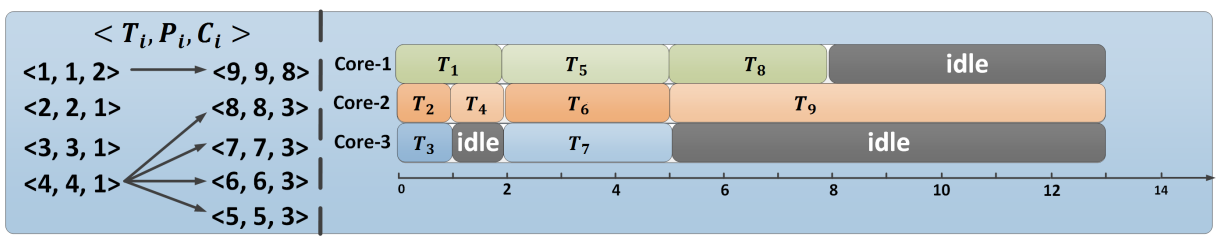

(a) Execution time reduced: $C_{i}^{\prime}=C_{i}-1$

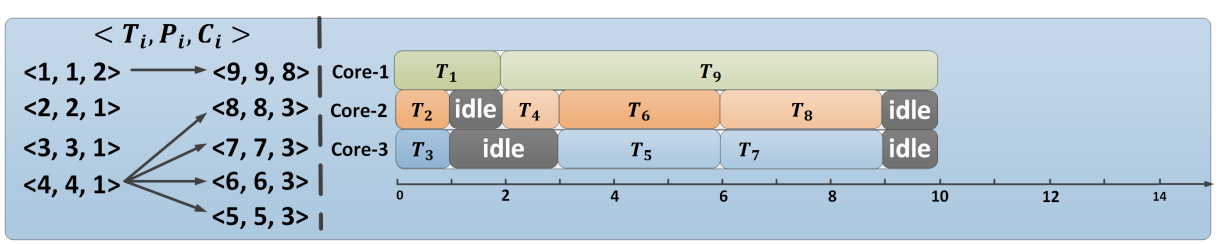

(b) Execution time reduced, while execute in the same order as Fig 4 (a)

Figure 5: Scheduling anomaly while reducing the execution time

dynamically in the multicore platform. As shown in Fig 5 (a), the "besteffort" scheduler chooses to execute task $T_{4}$ when processor Core- 2 is free, leading to the change of execution order compared with the execution order of Fig 4 (a), and the scheduling length of Fig.5 (a) increases. Third, the execution order can be changed when trying to avoid hazards with mutexes, which are caused by tasks that share the same resource. For example, the key idea (e.g., the Priority Ceiling Protocol and the Priority Inheritance Protocol) to overcome deadlock and priority inversion problem is to adjust the execution order of tasks that acquire the same resource lock [18, 50]. However, the adjustment leads to the change of execution order, and reduces the predictability of the task execution in turn.

In summary, both the I/O behavior and the function task must be predictable in CPS. Both of them must have predictable time property and predictable order property.

\section{Define Predictability for CPS}

According to [4, 21], the notion of predictability should capture if, and to what level of precision, a specified property of a system can be predicted by a system-specific optimal analysis. To enable a concise and uniform description of predictability instances, Grund proposes a template for the definition of predictability, which should consist of three aspects: 1) property to be 
predicted; 2) sources of uncertainty, and; 3) quality measure [21]. In this section, we analyze the sources of uncertainties of different properties, and use the template and the uncertainties to define the predictability of different properties that we summarized in the last section.

\subsection{Predictability of Input Behavior}

In this section, we analyze the sources of uncertainties of different kinds of inputs, and propose definitions for their predictability. According to the definition of input (Section 2.2), we classify the input into sensor and network. Since the uncertainties of network is more significant than that of sensors, we classify the sensor inputs, whose values are transmitted through network, into the network inputs.

\subsubsection{Time Predictability of Sensor Input}

The sensor changes its output state after a period of time delay when an input parameter changes. The delay includes the conversion time of the A/D converter and further time delays due to signal processing, for example, filter operation, outlier detection [1]. The time required for a sensor to change from its previous state to a final settled value within a tolerance band of the correct new value is called sensor response time [15, 55]. The sensor response time changes dynamically because it can be affected by many factors, such as the workload, construction details, properties and geometries of the sensor's internal material, and the properties of the medium surrounding the sensor.

We use the variability of the sensor response time to measure the ready time predictability of the sensor input. Let $I$ and $S$ denote all the possible inputs and states of the sensor. The ready time predictability of the sensor input is defined as:

$$
\operatorname{Pr}_{\text {sensor }}(I, S):=\min _{i_{1}, i_{2} \in\left[1, N_{I}\right]} \min _{j_{1}, j_{2} \in\left[1, N_{S}\right]} \frac{T_{r}\left(I_{i_{1}}, S_{j_{1}}\right)}{T_{r}\left(I_{i_{2}}, S_{j_{2}}\right)}
$$

where $I_{i_{1}}, I_{i_{2}} \in I$ and $S_{j_{1}}, S_{j_{2}} \in S . \quad N_{I}$ and $N_{S}$ are the numbers of sensor inputs $I$ and sensor states $S$, which are determined theoretically. $T_{r}\left(I_{i}, S_{j}\right)$ is used to calculate the sensor response time when the sensor input parameter is $I_{i}$ and the sensor is at the $S_{j}$ state. $T_{r}$ can be calculated through theoretical approaches [26]. $T_{r}$ can also be determined by the combination of theoretical approaches and experiment approaches, because properties that affect the sensor response time are not known thoroughly and may change under 


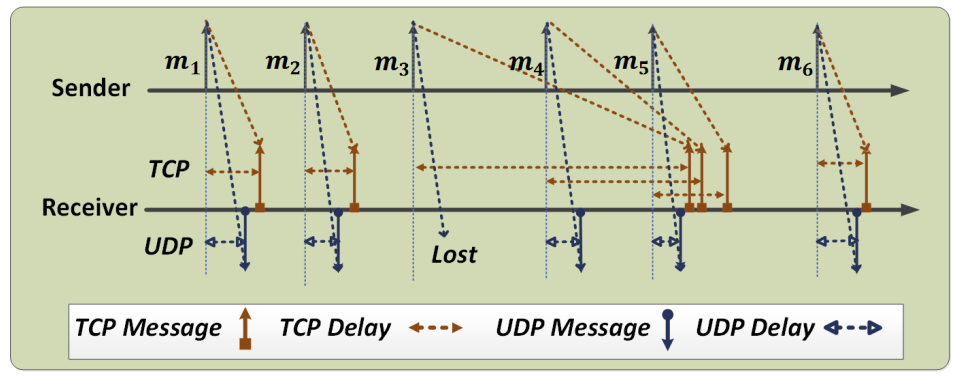

(a) Event-Triggered Communication

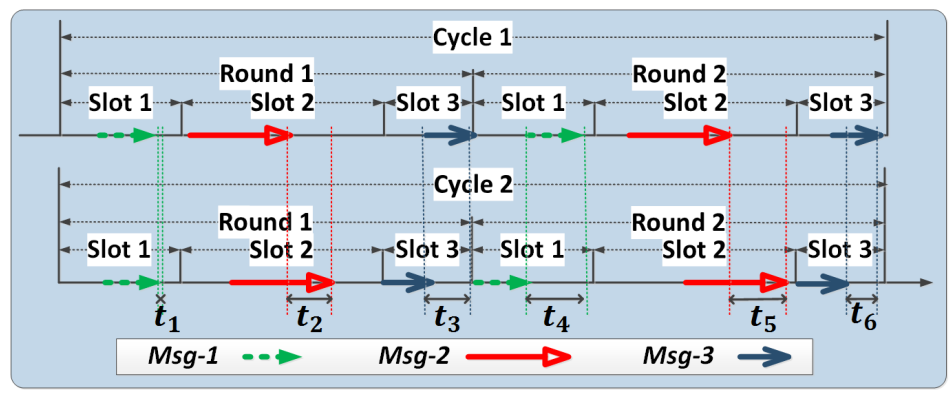

(b) Time-Triggered Communication

Figure 6: Event-Triggered and Time-Triggered Communication Jitter

process operating or aging conditions [24, 51]. The quotient is the quality measure: $P r_{\text {sensor }} \in(0,1]$, where the lager $P r_{\text {sensor }}$ is, the more predictable the ready time of the sensor input is. If $\operatorname{Pr}_{\text {sensor }}$ is close to 1 , the sensor is slightly affected by its environments and the variability of the sensor response time is small. In this case, the time to process the input can be set compactly, so that the values in the input port can be processed as soon as possible. This can improve the predictability of the input-determined programs. In addition, this can reduce the time to use buffers, which can contribute to the predictability of the runtime systems.

\subsubsection{Time Predictability of Network Input}

From the temporal point of view, communication services are classified into Event-Triggered (ET) communication and Time-Triggered (TT) communication [35]. In this section, we analyze the time uncertainties of the ET communication and TT communication, respectively, and propose metrics to define the ready time predictability of the network input.

In an Event-Triggered communication system, a sender sends a message 
whenever a significant event occurs at the sender, and places the message in a queue at the senders site until the basic message transport service is ready to transport the message to the receiver. After arrival of the message at the receiver, the message is placed in a receiver queue until the receiver consumes the message. Typical ET communication protocols include Ethernet protocol [46], Controller Area Network (CAN) protocol [13], and User Datagram Protocol (UDP) protocol from the Internet suite protocol. The delay of the ET communication protocols can be affected by the bus workload, the message priority, the data rate and so on [37]. We take the TCP and UDP for example. As shown in Fig.6 (a), messages arrive through a TCP channel experience different delays though they are received in the right order. The delays of different messages vary greatly. No messages are lost during the transmission. If the messages are transmitted through a UDP channel, though the delays are smaller, some messages are lost. Other ET communication protocols try to reduce the loss or the delay. However, protocols preventing loss do so by re-transmission which creates variable delay or jitter, one cannot eliminate both [29]. The network jitter caused by the variability of the delays seriously reduce the predictability of ET real-time networks. Therefore, the influence of the network jitter on the predictability of network inputs needs to be measured.

In a Time-Triggered communication system, the sender and receiver(s) agree a priori on a cyclic time-controlled conflict-free communication schedule for the sending of time-triggered messages [35]. Typical TT communication protocols include Time-Triggered protocol (TTP) [36], Time-Triggered Ethernet (TTEthernet) [34], and FlexRay [9]. Even though the TT communication is claimed to be predictable, the reality is not as predictable as what the TT communication claims. The first and foremost thing that affects the predictability of the TT communication protocol is the synchronization error [37]. In addition, the predictability can also be affected by technique details. We take the TTP for example. TTP uses the message description list (MEDL), which is constructed off-line and loaded on each TTP controller before operation starts, to specify which node will transmit which message at what time. The MEDL describes the operation of the bus on a common global timeline, which is produced by the clock synchronization protocol. Time is divided into cycles, rounds, and slots (Fig.6(b)). Each cycle contains a number of rounds and each round a number of slots. Cycles are repeated as long as the system runs, in exactly the same way. Rounds have the same duration, whereas slots within a round may have different durations. Each slot 
is assigned to a node, which transmits exclusively during that slot. Within a slot, a node transmits a frame, which contains one or more messages. We assume that the number of messages of each frame is the same. The TTP guarantees that (only) one node sends a frame in its slot. However, the starting and ending times of transmission are not exactly the same in each slot of different rounds and different cycles. In Fig.6(b), $t_{i}$ is the transmission ending time variances between different slots of different rounds. Even though the time variances of the TT communication protocols are smaller than that of the ET communication protocols in most cases, the variances should be carefully measured to assess their influences on the predictability of the TT communication protocols for the time safety critical CPS.

We use the the network delay to measure the time predictability of the network inputs. We assume that there are $M$ flows of messages need to be transmitted over the network. The delay set of all the messages is $D$, which can be analyzed statically. $T_{d}$ is the delay of a flow of message. The predictability of network input is defined as:

$$
\operatorname{Pr}_{\text {network }}(D):=\min _{1 \leq i, j \leq M} \frac{T_{d}\left(J T_{i}, L D_{i}, S D_{i}, W D_{i}\right)}{T_{d}\left(J T_{j}, L D_{j}, S D_{j}, W D_{j}\right)}
$$

where $T_{d}(J T, L D, S D, W D)$ is used to calculate the network input delay. $J T_{i}$ is the release jitter of the $i_{t h}$ flow of message. $L D_{i}$ is the transmission time on links along the considered path. $S D_{i}$ is the delay caused by switching latency. $W D_{i}$ is the waiting delay caused by competitions with other frames in the output buffers. The quantification over these four kinds of delays in $T_{d}$ capture the uncertainties of network delay. The way $T_{d}$ is calculated is highly dependent on the model of the network and the environments [3, 6, 17, 44, 49, 57, 58. The quotient is the quality measure: $\operatorname{Pr}_{\text {network }} \in(0,1]$, where the larger $P r_{n e t w o r k}$ is, the more predictable the network input is. The network are the major sources of input uncertainties, therefore, if $P r_{\text {network }}$ is close to 1 , the predictability of the inputs can be improved significantly, which in turn increases the predictability of the system.

\subsubsection{Ready Order Predictability of Inputs Set}

In this section, we introduce a method to determine all the possible ready orders based on the ready times of different inputs at first. Then, we propose a method to calculate the buffer costs of the inputs ready order. In the end, we define the predictability of the inputs ready order based on buffer costs. 


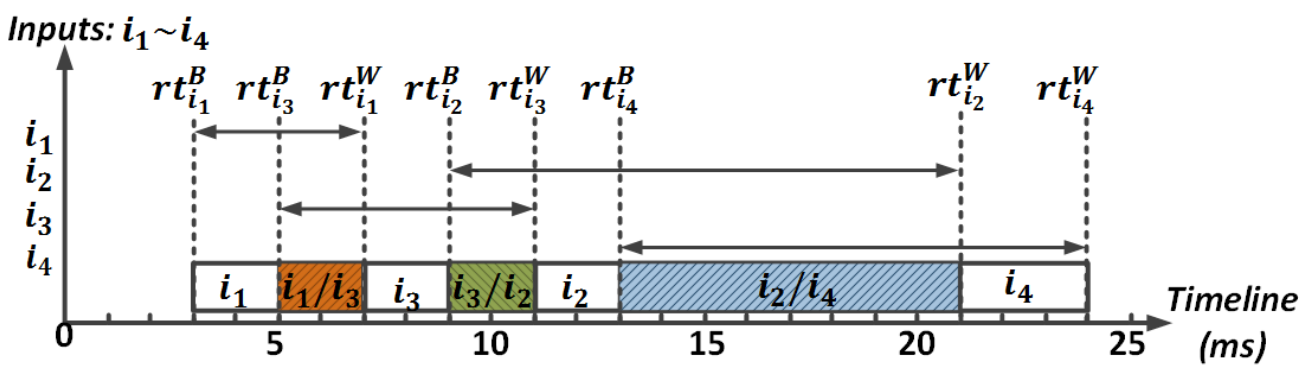

Figure 7: Best Case and Worst Case Ready Times of different Inputs in Fig2

Find All the Possible Input Ready Orders. Assume that $R T_{I}$ is the set of possible ready times of an inputs set. $R T_{I}=\left\{R T_{i} \mid i \in I\right\}$, where $I$ is the input set and $i$ is an input of $I . R T_{i}$ is the set of response times of input $i$. $R T_{i}=\left\{r t \mid r t_{i}^{B} \leq r t \leq r t_{i}^{W}, r t_{i} \in \mathbb{R}\right\}$, where $r t_{i}^{B}$ and $r t_{i}^{W}$ are the Best Case Ready Time and the Worst Case Ready Time of input $i$, respectively. We introduce Theory 1 to limit the ready orders of $I$.

Theory 1. $\forall i, j \in I$, if $r t_{i}^{W}<r t_{j}^{B}$, then $R T_{i} \cap R T_{j}=\Phi$, and input $i$ becomes ready earlier than input $j$, there must exist $O_{i}<O_{j}$, which means that input $i$ must be at the front of input $j$ in the inputs ready order.

The ready order of $I$ cannot violate the order limited by Theory 1 . Therefore, the problem to find the possible ready orders of $I$ can be solved by finding all the possible orders of a partially ordered sequence of vertexes. We use the Topological Sorting to find all the possible input ready orders [14]. We use $R T_{I}$ to construct the Directed Acyclic Graph (DAG), i.e., G. Let $G=(I, E)$, where $I$ is the inputs set of the actor, and $E$ is the set of directed edges generated according to the following rule: $\forall i, j \in I$, if $O_{i}<O_{j}$, add an directed edge from vertex $i$ to vertex $j$. The possible orders can be found by algorithms shown in [32], which can generate all the possible solutions for a topological sort.

We take Fig 7 for example. Assume that $r t_{i_{1}}^{B}=3, r t_{i_{1}}^{W}=7 ; r t_{i_{2}}^{B}=9, r t_{i_{2}}^{W}=$ $21 ; r t_{i_{3}}^{B}=5, r t_{i_{3}}^{W}=11 ; r t_{i_{4}}^{B}=13, r t_{i_{4}}^{W}=24(\mathrm{~ms})$. According to Theory 1 , the orders that the inputs become ready must satisfy the following rules: $O_{i_{1}}<$ $O_{i_{2}}, O_{i_{1}}<O_{i_{4}}$, and $O_{i_{3}}<O_{i_{4}}$. We use the above rules and $I$ to construct the DAG. $i_{1}, i_{2}, i_{3}$, and $i_{4}$ are the vertexes of the DAG. Since $O_{i_{1}}<O_{i_{2}}$, a directed edge is added from $i_{1}$ to $i_{2}$. The same goes for the directed edge 


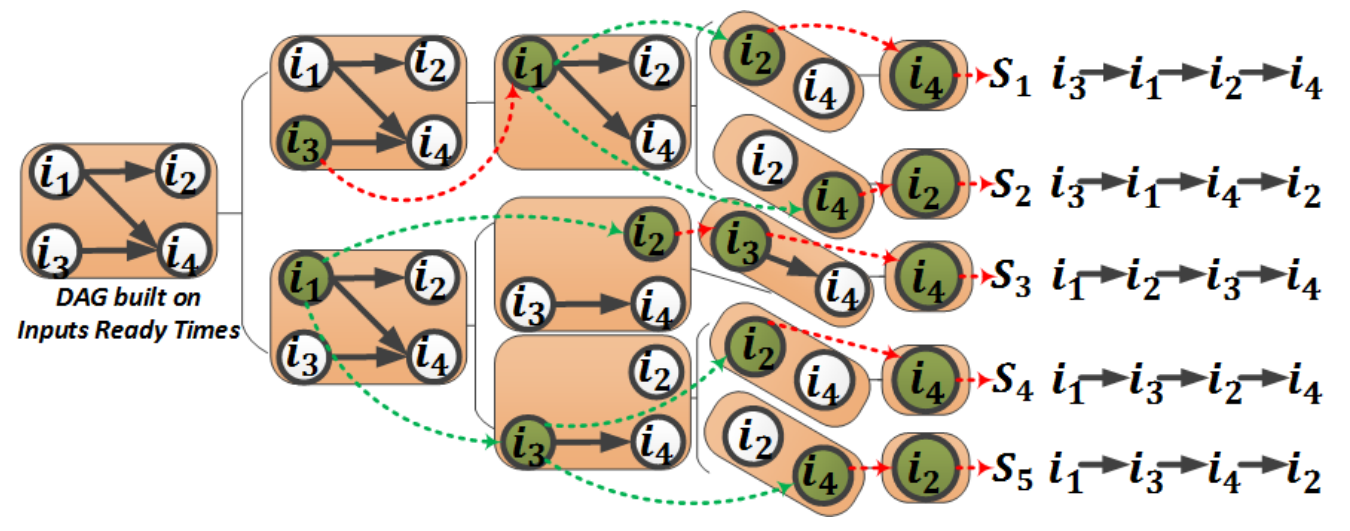

Figure 8: Find All the Possible Input Ready Orders by the Topological Sorting Algorithm

from $i_{1}$ to $i_{4}$ and the edge form $i_{3}$ to $i_{4}$. Fig 8 shows the DAG built on $R T_{I}$ and the way to find all the possible inputs ready orders. According to algorithms proposed in [32], all the possible ready orders are as following: $S_{1}: i_{3} \rightarrow i_{1} \rightarrow i_{2} \rightarrow i_{4}, S_{2}: i_{3} \rightarrow i_{1} \rightarrow i_{4} \rightarrow i_{2}, S_{3}: i_{1} \rightarrow i_{2} \rightarrow i_{3} \rightarrow i_{4}, S_{4}:$ $i_{1} \rightarrow i_{3} \rightarrow i_{2} \rightarrow i_{4}$, and $S_{5}: i_{1} \rightarrow i_{3} \rightarrow i_{4} \rightarrow i_{2}$.

Calculate the Buffer Costs of Each Inputs Ready Order. The buffer cost represents the influence of the buffer to the system's predictability. It is not the number of buffers, because the buffer affects the system's predictability as long as it exists [54]. Therefore, we use a buffer's accumulated influence on the system to measure the buffer cost. Assume that the cost of each buffer operation is the same, the buffer cost is defined as follows: if a number of $n$ inputs values are buffered while reading input $i$, the buffer cost while reading input $i$ is $n$. The buffer costs of the inputs ready order is the sum of buffer costs while reading all the inputs.

We take the ready order $S_{2}$ (shown in Fig 8 ) for example. The ready order of $S_{2}$ is $i_{3} \rightarrow i_{1} \rightarrow i_{4} \rightarrow i_{2}$. The buffer costs that are needed to process $S_{2}$ is calculated in the way shown in Fig.9. At the $1^{\text {st }}$ time instant, the actor needs to read $i_{1}$, which is not ready yet. However, since $i_{3}$ is ready now, one buffer cost is required to store its value. At the $2^{\text {nd }}$ time instant, the actor reads $i_{1}$ again, which is ready now. Since $i_{3}$ is still in the buffer, one buffer cost is needed to maintain the buffer. At the $3^{r d}$ time instant, the actor reads $i_{2}$, which is not ready yet. Since $i_{4}$ becomes ready at this instant, a new buffer is required. $i_{3}$ is till in the buffer, therefore two buffer costs are needed at this instant. At the $4^{\text {th }}$ time instant, actor reads $i_{2}$, which becomes ready just in 


\begin{tabular}{c|ccccc}
$\begin{array}{c}\text { Time } \\
\text { Instants }\end{array}$ & Read & \multicolumn{3}{c}{ Inputs } & $\begin{array}{c}\text { Buffer } \\
\text { Costs }\end{array}$ \\
\hline \hline 1 & $\boldsymbol{i}_{1} \times$ & $\boldsymbol{i}_{3}$ & $\boldsymbol{i}_{1}, \boldsymbol{i}_{2}, \boldsymbol{i}_{4}$ & $\boldsymbol{i}_{3}$ & 1 \\
2 & $\boldsymbol{i}_{1} \checkmark$ & $\boldsymbol{i}_{3}, \boldsymbol{i}_{1}$ & $\boldsymbol{i}_{2}, \boldsymbol{i}_{4}$ & $\boldsymbol{i}_{3}$ & 1 \\
3 & $\boldsymbol{i}_{2} \times$ & $\boldsymbol{i}_{3}, \boldsymbol{i}_{4}$ & $\boldsymbol{i}_{2}$ & $\boldsymbol{i}_{3}, \boldsymbol{i}_{4}$ & 2 \\
4 & $\boldsymbol{i}_{2} \checkmark$ & $\boldsymbol{i}_{3}, \boldsymbol{i}_{4}, \boldsymbol{i}_{2}$ & $\mathrm{NULL}$ & $\boldsymbol{i}_{3}, \boldsymbol{i}_{4}$ & 2 \\
5 & $\boldsymbol{i}_{3} \checkmark$ & $\boldsymbol{i}_{3}, \boldsymbol{i}_{4}$ & NULL & $\boldsymbol{i}_{4}$ & 1 \\
6 & $\boldsymbol{i}_{4} \checkmark$ & $\boldsymbol{i}_{4}$ & NULL & NULL & 0 \\
\hline
\end{tabular}

Figure 9: Calculate the Buffer Costs of $S_{2}: i_{3} \rightarrow i_{1} \rightarrow i_{4} \rightarrow i_{2}$

time. Therefore, two buffer costs are in need to maintain the buffers which are used by $i_{3}$ and $i_{4}$. At the $5^{\text {th }}$ time instant, actor reads $i_{3}$, and releases the buffer for $i_{3}$. Therefore, only the buffer cost for $i_{4}$ is in need. At the $6^{\text {th }}$ time instant, actor reads $i_{4}$, and all the buffers are released. No buffer costs are in need at this instant. Therefore, if the inputs become ready in the $S_{2}$ order, the buffer costs is the sum of the costs in each time period, namely, 7 .

In the following, we present a generalized method to calculate the buffer costs of a determined inputs ready order. Let $I$ denote the set of all the $N$ inputs. The states of $I$ are stored in the ready states matrix, $S_{r}=$ $\left[s_{1}, s_{2}, \ldots, s_{j}, \ldots, s_{N}\right]^{\mathrm{T}}$, where $s_{j}=\left[s_{i, 1}, s_{i, 2}, \ldots, s_{i, j}, \ldots, s_{i, N}\right] . s_{j}$ is a sequence of input ready states when the $j_{t h}$ input becomes ready. If input $i_{k}$ of $s_{j}$ is ready, then $s_{j, k}=1$, or $s_{j, k}=0$. The ready states matrix of $S_{2}(\mathrm{Fig}, 8)$ is shown as follows:

$$
S_{r}=\begin{gathered}
s_{1} \\
s_{2} \\
\vdots \\
s_{N}
\end{gathered}\left(\begin{array}{cccc}
i_{1} & i_{2} & \cdots & i_{N} \\
s_{1,1} & s_{1,2} & \cdots & s_{1, N} \\
s_{2,1} & s_{2,2} & \cdots & s_{2, N} \\
\vdots & \vdots & \ddots & \vdots \\
s_{N, 1} & s_{N, 2} & \cdots & s_{N, N}
\end{array}\right), S_{2}=\left(\begin{array}{cccc}
0 & 0 & 1 & 0 \\
1 & 0 & 1 & 0 \\
1 & 0 & 1 & 1 \\
1 & 1 & 1 & 1
\end{array}\right)
$$

We propose Alg 1 to calculate the buffer costs of the inputs ready order. Given the states matrix $S_{r}$, the number of inputs $N$, assume that the inputs are read from the $1^{\text {st }}$ input to the $N^{\text {th }}$ input in monotonous order. Alg.1 simulates the input reading process while the ready order is $S_{r}$. The value of $i$ in Alg. 1 is the index of the time instants, and read is used to record which 
input should be read at this instant. Codes between line 5 and line 8 are used to change the input state if the input is ready and has been read. Codes between line 9 and line 11 are used to calculate the buffer costs. In the worst case, in which the inputs become ready in the inverse order compared with the order which is specified in the programs, the outer loop executes $2 \cdot N$ times. In this case, the codes between line 5 and line 8 execute $(N+1) N / 2+N$ times, and codes between line 9 and line 11 execute $2 N^{2}$ times. Therefore, the worst case time complexity of $\operatorname{Alg} 1$ is $((N+1) N / 2+N)+2 N^{2}$, which equals to $O\left(N^{2}\right)$, where $N$ is the number of inputs.

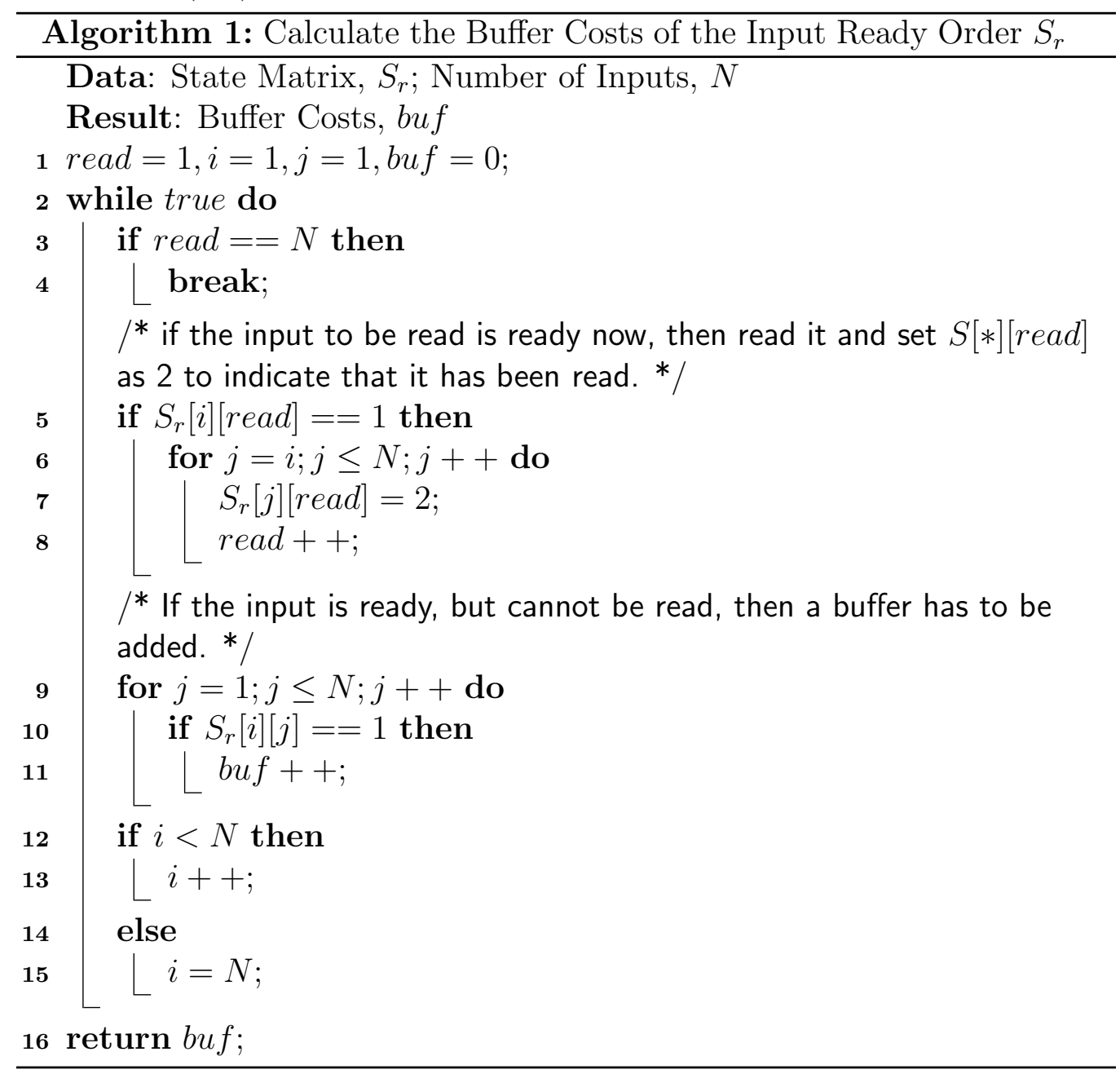

Define the Predictability of the Inputs Ready Order. Based on Alg,1, we can calculate the buffer costs of every ready order shown in Fig.8. The buffer 
costs of $S_{1}, S_{2}, S_{3}, S_{4}$, and $S_{5}$ are $4,7,0,3$, and 6, respectively. We can see that the buffer costs vary greatly among different ready orders. Since the buffer costs significantly affect the predictability of the system [33, 54], we use the quotient of the minimum over the maximum buffer costs to measure the predictability of the inputs order.

Let $S$ denote the set of inputs ready orders that are calculated through the way shown in Fig8. Assume that $B u f_{S_{i}}$ is the buffer costs of $S_{i}$, which is calculated by $\operatorname{Alg}$. 1 . If $\exists S_{i} \in S \wedge B u f_{S_{i}}=0, S$ is refined as $\widehat{S}=$ $\left\{S_{r} \mid S_{r} \in S \wedge B u f_{S_{i}} \neq 0\right\}$, because the zero buffer cost does not affect the system's predictability. If the cardinality of $\widehat{S}$ is zero, the ready order predictability is 1 , else the input ready order predictability is defined as:

$$
\operatorname{Pr}_{\text {InputsOrder }}(\widehat{S}):=\min _{S_{i}, S_{j} \in \widehat{S}} \frac{B u f_{S_{i}}}{B u f_{S_{j}}}
$$

The quotient is the quality measure: $\operatorname{Pr}$ InputsOrder $\in(0,1]$, where the larger $P r_{\text {InputsOrder }}$ is, the more predictable the inputs ready order is. However, $P r_{\text {InputsOrder }}=1$ does not necessarily mean that there is only one order in $S$, because some different orders may need the same buffer costs, in which case we think that these orders affect the predictability of the system at the same level. A perfect predictable input ready order does not necessarily mean that the inputs become ready in the ideal order every time. Rather, it emphasizes that the orders in which the inputs become ready are the same under different ready times of the inputs. In this case, since the buffer costs to read the inputs are the same, they have the same influence on the system. This is good for the predictability, because we can confidentially tell how the system is affected by the inputs, and try to fix the bad effects. More importantly, if $\mathrm{Pr}_{\text {InputsOrder }}$ is close to 1, the input-determined programs have more predictable behaviors, and more reliable composition and verification results [40]. Otherwise, if $P r_{\text {InputsOrder }}$ is close to 0 , the predictability of the input-determined programs and the reliability of the composition and verification results are badly affected by the inputs ready order. In this case, the calculation and definition in this section can help to find the bottleneck and provide ideas to improve the predictability, e.g., reducing the number of ready time intersections shown in Fig.7. 


\subsubsection{Predictability of Inputs Set}

Compared with the network delay, the delay of sensor is shorter in most cases. However, that does not mean that the predictability of network is more important than that of the sensor to CPS, since any delay that outstrips the predictability may lead to the disaster of the time critical CPS. Therefore, the predictability of the inputs set depends on the factor who has the worst predictability. Taking the inputs ready order into consideration, the predictability of the inputs set is defined as:

Definition 1. Given a platform $\mathcal{P}$, and its input set $\mathcal{I}$. Its sensor inputs set is $\mathcal{I}_{S}$, and its network inputs set is $\mathcal{I}_{N}$. The predictability of input set $\mathcal{I}$ is:

$$
\operatorname{Pr}_{\mathcal{I}}:=\min \left(\min _{i_{s} \in \mathcal{I}_{S}}\left(\operatorname{Pr}_{i_{s}}\right), \min _{i_{n} \in \mathcal{I}_{N}}\left(\operatorname{Pr}_{i_{n}}\right), \operatorname{Pr} r_{\text {InputsOrder }}\right)
$$

where $P r_{i_{s}}$ is the predictability of sensor input $i_{s}$ (Equation 1), $P r_{i_{n}}$ is the predictability of network input $i_{n}$ (Equation 2), and $P r_{\text {InputsOrder }}$ is the predictability of inputs ready order of $\mathcal{I}$ (Equation 3). If $P r_{\mathcal{I}}$ is close to 1, the inputs are highly predictable, and the system tends to has more predictable behaviors and reliable verification results, and can be composed more easily. However, if $P r_{\mathcal{I}}$ is close to 0 , the inputs are badly predictable. In this case, the testing and verification of CPS are very hard and unreliable, and it is very hard to ensure the safety of CPS. We need to figure out the critical factor that depresses the input predictability according to Equation 4, and spare no efforts to improve the predictability of that factor.

\subsection{Predictability of Output Ports}

According to [31], output is defined as writing the results of the computation to one or more nodes, which may be devices and/or main memory. This section discusses the sources of uncertainties of writing outputs, and proposes definitions for the output predictability.

\subsubsection{Time Predictability of Local Output}

If the output of a task is only used by other tasks in the same node, the output is called local output, whose value should be written back into memory for the usage of other tasks. For example, in Fig, 2, task $C_{2}$ depends on the computation result of task $C_{1}$, to ensure the consistency of the memory system, $C_{2}$ will wait until $C_{1}$ finishes writing $s_{1}$ into the main memory. Since only the value of the local platform is updated, the unpredictability 
of the local output mainly comes from the memory access latency [56]. We use the latency of updating the value in main memory to define the time predictability of the local outputs:

Definition 2. In platform $\mathcal{P}$, the latency to update the memory value is $l_{c h}$ if all caches hit, and the latency is $l_{c m}$ if all caches miss. Once starting to write output, the time predictability of the local output is:

$$
\operatorname{Pr}_{\text {LocalOut }}:=\frac{l_{c h}}{l_{c m}}
$$

The quotient is the quality measure: $P r_{\text {LocalOut }} \in(0,1]$. It is the quotient of the latency when cache hit over the latency when cache miss to update memory value. However, the reality would be much more complicated because of the great uncertainty of the memory system. For example, when the processor is ready to write memory, the updating job has to wait if the main memory is refreshing, leading to the unpredictability of the output. The predictability of the memory system can be improved by adopting the scratchpad memory [5, 45]. Since the local output of the precedent task is used as the input of the subsequent task in the same node, therefore, if $P r_{\text {LocalOut }}$ is close to 1, the predictability of the subsequent task is improved significantly. In addition, a highly predictable local output can reduce the data synchronization cost between the precedent task and the subsequent task, and thus increase the predictability and reliability of the system.

\subsubsection{Time Predictability of Network Output}

If the output value needs to be broadcasted to other $\operatorname{sink}(\mathrm{s})$ through network, the output is called as network output, whose unpredictability is mainly caused by the variability of network delays of different receivers. Different from the network input, the network output have to update values in more than one remote receivers. Therefore, the time predictability of network outputs can be measured by the variability of the intervals between the earliest time and the latest time when the sinks receive the message:

Definition 3. Given a platform $\mathcal{P}$, and its sender $S$. The receiver set is $\mathcal{R}$, which has $N$ receivers. A number of $M$ flows of messages need to be sent to each receiver. The delay of transmission to $\mathcal{R}$ is $\mathcal{D}$. The time predictability 
of network outputs of platform $\mathcal{P}$ is:

$$
\operatorname{Pr}_{\text {NetOut }}(\mathcal{R}):=\min _{1 \leq i_{1}, i_{2} \leq N} \min _{1 \leq j_{1}, j_{2} \leq M} \frac{T_{d}\left(J T_{j_{1}}^{i_{1}}, L D_{j_{1}}^{i_{1}}, S D_{j_{1}}^{i_{1}}, W D_{j_{1}}^{i_{1}}\right)}{T_{d}\left(J T_{j_{2}}^{i_{2}}, L D_{j_{2}}^{i_{2}}, S D_{j_{2}}^{i_{2}}, W D_{j_{2}}^{i_{2}}\right)}
$$

where $T_{d}\left(J T_{j}^{i}, L D_{j}^{i}, S D_{j}^{i}, W D_{j}^{i}\right)$ is used to calculate the latency of the $j^{\text {th }}$ flow of messages which are sent to the $i^{\text {th }}$ receiver. JT, $L D, S D$, and $W D$ are the four kinds of network delays which are used to capture the network uncertainty (Section 3.1.2). The property to predict is the network delay to of different messages to different receivers. Definition 3 can also be used to measure the network output which has only one remote receiver. In this case, $N$ is 1 , and $P r_{\text {NetOut }}$ is the quotient of the best case delay over the worst case delay to the remote receiver. The quotient is the quality measure of the predictability of the network output: $\operatorname{Pr}_{\text {NetOut }} \in(0,1]$, where the larger $P r_{\text {NetOut }}$ is, the better the time predictability of the network output is. If $\operatorname{Pr}_{\text {NetOut }}$ is close to 0, we must find out the receiver which has the worst predictability according to Equation 6, and improve the predictability of the network transmission to that receiver.

\subsection{Predictability of Function Tasks}

Considering the concurrent and preemptive characteristics of CPS, we propose to use the task response time to define the time predictability of function tasks in this section. We define the order predictability of tasks set based on the distance between the actual execution order and the ideal execution order. Tasks discussed in this section are Preemptive, Non-Migratable, and Fix-Priority $(\mathrm{P} / \mathrm{N} / \mathrm{F})$ tasks.

\subsubsection{Time Predictability of Function Tasks}

Due to the multitasking, high concurrency, and frequently preemptive characteristics of CPS, the measure of the time predictability of function tasks should take the interferences from other tasks into consideration. The task response time is defined as the time between the release of a task and its corresponding completion [10]. The estimation of response time includes the execution time, the preemption of other tasks, and interferences from OS kernel. Therefore, we use the response time, i.e., Best Case Response Time (BCRT) and Worst Case Response Time (WCRT) to define and measure the time predictability of individual function task and tasks set. 
Calculation of WCRT and BCRT. Burns proposed the methods to estimate the WCRT of a task in the preemptive priority based scheduling algorithms 11. Task $i$ is assumed to consist of an infinite number of invocation requests, each separated by $T_{i}$, which is the minimum inter-arrival time for the events that releases the task. Each invocation of task $i$ requires $W C_{i}$ computation time in the worst case. During this time, the task cannot be preempted. A ceiling priority protocol is used to solve the priority inversion problem, which gives rise to the blocking time. $W B_{i}$ is the maximum time task $i$ can be blocked while waiting for a lower priority task. The WCRT of task $i$ is calculated as [11]:

$$
W R_{i}=W B_{i}+W C_{i}+\sum_{j \in h p(i)}\left\lceil\frac{W R_{i}}{T_{j}}\right\rceil W C_{j}+\left\lceil\frac{W R_{i}}{T_{c l k}}\right\rceil C_{c l k}+\sum_{f \in f p t}\left\lceil\frac{W R_{i}}{T_{f}}\right\rceil C_{p e r}
$$

where $W R_{i}$ is the WCRT of task $i$, and $h p(i)$ is the set of tasks who have higher priorities than task $i$. Assume that tasks are on the delay queue but none are removed, $C_{c l k}$ is the overhead occurring on each interrupt, $C_{p e r}$ is the cost of moving one task from the delay-queue to run-queue, and $f p t$ is the set of fictitious periodic tasks, which have the same period with task $i$. $W R_{i}$ can be calculated through the iterative process as explained in [11].

The calculation of BCRT is researched in [10, 48], which take account of the preemptions of higher priority tasks. However, as long as there are preemptions, the runtime overheads (e.g., removing tasks from delay-queue to run-queue) are unavoidable. Therefore, the runtime system overheads should also be considered when calculating the BCRT. The BCRT is calculated in the following way:

$$
B R_{i}=B B_{i}+B C_{i}+\sum_{j \in h p(i)}\left\lceil\frac{B R_{i}}{T_{j}}\right\rceil B C_{j}+\left\lceil\frac{B R_{i}}{T_{c l k}}\right\rceil C_{c l k}+\sum_{f \in f p t}\left\lceil\frac{B R_{i}}{T_{f}}\right\rceil C_{p e r}
$$

$B R_{i}$ is the best case response time of task $i . B C_{i}$ is the best case computation time of task $i$, and $B B_{i}$ is the minimum time task $i$ can be blocked. The other parameters of Equation 8 have the same meaning with that of Equation 7. $\left\lceil\frac{B R_{i}}{T_{c l k}}\right\rceil C_{c l k}$ and $\sum_{f \in f p t}\left\lceil\frac{B R_{i}}{T_{f}}\right\rceil C_{p e r}$ take the runtime overheads and interruptions of other tasks into consideration, respectively.

It should be noted that methods to calculate BCRT and WCRT vary greatly in different situations, such as different scheduling algorithms. In this paper, we use the classical methods to show the basic ideas and the sources of uncertainties of the response time. However, the specific methods to estimate BCRT and WCRT should be determined by the characteristics of the tasks and their execution environments. 
Time Predictability of Individual Task. We use the quotient of the BCRT over the WCRT of an individual task to define its time predictability.

Definition 4. Let $\mathcal{T}$ denote the tasks set, whose tasks can be executed concurrently. For any task $\tau_{i} \in \mathcal{T}$, assume that its BCRT is $B R_{\tau_{i}}$, and its $W C R T$ is $W R_{\tau_{i}}$. The time predictability of task $\tau_{i}$ is:

$$
\operatorname{Pr}_{\tau_{i}}:=\frac{B R_{\tau_{i}}}{W R_{\tau_{i}}}
$$

where $B R_{\tau_{i}}$ and $W R_{\tau_{i}}$ are calculated through Equation (8) and Equation (7), respectively. The quotient is the quality measure: $P r_{\tau_{i}} \in(0,1]$, where the higher $P r_{\tau_{i}}$ is, the more predictable task $\tau_{i}$ is. If the $P r_{\tau_{i}}$ is close to zero, the time predictability of task $\tau_{i}$ is highly unpredictable. Since Definition 4 captures the sources of uncertainties of response time, we can use it to find the critical uncertainty sources and improve its time predictability. Compared with the definitions in [21, 28, 53, Definition 4 introduces the interferences from other tasks and overheads of the runtime systems. Therefore, Definition 4 is more suitable and practical for the concurrent and preemptive systems.

Time Predictability of Tasks Set. The time predictability of tasks set is affected by various factors, e.g., the task characteristic, runtime system, and scheduler [47]. Since the response time reflects the influences of those factors, we use the response time to define the time predictability of tasks set.

Definition 5. Let $\mathcal{T}$ denote the tasks set, whose tasks can be executed concurrently. For any task $\tau_{i} \in \mathcal{T}$, assume that its BCRT is $B R_{\tau_{i}}$, and its $W C R T$ is $W R_{\tau_{i}}$. The time predictability of tasks set $\mathcal{T}$ is:

$$
\operatorname{Pr}_{\mathcal{T}}(\mathcal{T}):=\frac{\max _{\tau_{i} \in \mathcal{T}}\left(B R_{\tau_{i}}\right)}{\max _{\tau_{j} \in \mathcal{T}}\left(W R_{\tau_{j}}\right)}
$$

$\max _{\tau_{i} \in \mathcal{T}}\left(B R_{\tau_{i}}\right)$ and $\max _{\tau_{j} \in \mathcal{T}}\left(W R_{\tau_{j}}\right)$ are the earliest time and the latest time, at which all the tasks in $\mathcal{T}$ complete. Note that they are not necessarily equal to the BCRT and the WCRT of the lowest priority task, respectively. Their values depend on the scheduling algorithms, properties of the tasks, and how BCRT and WCRT are calculated. The quotient is the quality measure: $P r_{\mathcal{T}} \in(0,1]$. The smaller $P r_{\mathcal{T}}$ is, the worse the time predictability of the 
tasks set is, and the harder it is to determine the periods and deadlines of $\mathcal{T}$. The designer has to face a dilemma: on the one hand, to guarantee the safety, choose a higher performance platform, which may run at lower utilization ratio because the tasks may completed earlier in most cases; on the other hand, to reduce the cost, set a risky deadline, which is not safe in the worst cases. If $P r_{\mathcal{T}}$ is close to 1 , which means that the completion time of the tasks set is highly predictable, the durations of this tasks set can be set compactly and safely with high confidence.

\subsubsection{Execution Order Predictability of Tasks Set}

In this section, we propose a method to describe the tasks execution order, and introduce the ideal execution order at first. Then, we introduce a theory to calculate the order distance between the actual execution order and the ideal execution order. At last, we use the variability of the execution order distances to define the predictability of tasks execution order.

Ideal Execution Order of Tasks Set. According to Theorem 4.2 of [22], for a priority-driven scheduling algorithm, the execution of tasks is predictable only if the priorities of independent tasks are assigned on the FIFO basis (i.e., the earlier the release time, the higher the priority). Therefore, we take the order in which tasks are released as the ideal execution order.

Given a set of $N$ tasks, denoted by $\mathcal{T}=\left\{\tau_{i} \mid i \in[1, N]\right\}$, where $\tau_{i}$ is a task. Let $T O I_{\mathcal{T}}$ denote the ideal execution order of the tasks in $\mathcal{T}$. $T O I_{\mathcal{T}}=$ $\left\{\tau_{1}^{\mathcal{I}}, \tau_{2}^{\mathcal{I}}, \ldots, \tau_{N}^{\mathcal{I}}\right\}$, where $\forall i, j \wedge i, j \in[1, N], \tau_{i}^{\mathcal{I}} \prec \tau_{j}^{\mathcal{I}}$, which means that $\tau_{i}$ is executed before $\tau_{j}$ when $i<j$. Since different tasks can be executed parallelly at the multicore platforms, the ideal execution order is refined as:

$$
\widehat{T O I_{\mathcal{T}}}=\{\underbrace{\left\{\tau_{1}^{\mathcal{I}}, \tau_{2}^{\mathcal{I}}, \ldots, \tau_{k_{1}}^{\mathcal{I}}\right\}}_{\tau_{1}^{\mathcal{I}^{\prime}}} \ldots, \underbrace{\left\{\tau_{k_{j-1}+1}^{\mathcal{I}}, \tau_{k_{j-1}+2}^{\mathcal{I}}, \ldots, \tau_{k_{j}}^{\mathcal{I}}\right\}}_{\tau_{j}^{\mathcal{I}^{\prime}}} \ldots, \underbrace{\left\{\tau_{k_{n-1}+1}^{\mathcal{I}}, \tau_{k_{n-1}+2}^{\mathcal{I}}, \ldots, \tau_{k_{n}}^{\mathcal{I}}\right\}}_{\tau_{n}^{\mathcal{I}^{\prime}}}\}
$$

where $n \leq N$, and $\tau_{j}^{\mathcal{I}^{\prime}} \in \widehat{T O I_{\mathcal{T}}}$. $\widehat{T O I_{\mathcal{T}}}$ is strictly partial ordered. $\widehat{T O I_{\mathcal{T}}}$ is the ideal execution order if it meets the following conditions: $\forall \tau_{i}, \tau_{j} \in$ $\mathcal{T}, \exists i^{\prime}, j^{\prime} \in[1, n] \wedge \tau_{i} \in \tau_{i^{\prime}}^{\mathcal{I}^{\prime}} \wedge \tau_{j} \in \tau_{j^{\prime}}^{\mathcal{I}^{\prime}}$, if $\tau_{i}$ is released before $\tau_{j}$, then $\tau_{i^{\prime}}^{\mathcal{I}^{\prime}} \prec \tau_{j^{\prime}}^{\mathcal{I}^{\prime}}$; if $\tau_{i}$ and $\tau_{j}$ are released at the same time, then $i^{\prime}=j^{\prime}$, namely, $\tau_{i}$ and $\tau_{j}$ are in the same element of $\widehat{T O I_{\mathcal{T}}}$. For example, we assume that the execution order shown in Fig 4 a) is the ideal execution order of the 9 tasks that we use in Fig 4 and Fig.5. Let $T$ denote the tasks set composed by the 9 tasks. 
The ideal execution order of $T$ is:

$$
\widehat{T O I_{T}}=\{\underbrace{\left\{T_{1}, T_{2}, T_{3}\right\}}_{\tau_{1}^{\mathcal{I}^{\prime}}}, \underbrace{\left\{T_{4}\right\}}_{\tau_{2}^{\mathcal{I}^{\prime}}}, \underbrace{\left\{T_{9}\right\}}_{\tau_{3}^{I^{\prime}}}, \underbrace{\left\{T_{5}, T_{6}\right\}}_{\tau_{4}^{\mathcal{I}^{\prime}}}, \underbrace{\left\{T_{7}, T_{8}\right\}}_{\tau_{5}^{\mathcal{I}^{\prime}}}\}
$$

The actual execution order of $\mathcal{T}$ is denoted by $T O A_{\mathcal{T}}=\left\{\tau_{1}^{\mathcal{A}}, \tau_{2}^{\mathcal{A}}, \ldots, \tau_{N}^{\mathcal{A}}\right\}$. It can be refined as $\widehat{T O A_{\mathcal{T}}}$ in the similar way with the refinement of $T O I_{\mathcal{T}}$. For example, the actual execution orders of Fig.5 a) and b) are:

$$
\begin{gathered}
\widehat{T O A_{T}^{a}}=\{\underbrace{\left\{T_{1}, T_{2}, T_{3}\right\}}_{\tau_{1}^{\mathcal{A}^{\prime}}}, \underbrace{\left\{T_{4}\right\}}_{\tau_{2}^{\mathcal{A}^{\prime}}}, \underbrace{\left\{T_{5}, T_{6}, T_{7}\right\}}_{\tau_{3}^{\mathcal{A}^{\prime}}}, \underbrace{\left\{T_{8}, T_{9}\right\}}_{\tau_{4}^{\mathcal{A}^{\prime}}}\} \\
\widehat{T O A_{T}^{b}}=\{\underbrace{\left\{T_{1}, T_{2}, T_{3}\right\}}_{\tau_{1}^{\mathcal{A}^{\prime}}}, \underbrace{\left\{T_{9}, T_{4}\right\}}_{\tau_{2}^{\mathcal{A}^{\prime}}}, \underbrace{\left\{T_{6}, T_{5}\right\}}_{\tau_{3}^{\mathcal{A}^{\prime}}}, \underbrace{\left\{T_{7}\right\}}_{\tau_{4}^{\mathcal{A}^{\prime}}}, \underbrace{\left\{T_{8}\right\}}_{\tau_{5}^{\mathcal{A}^{\prime}}}\}
\end{gathered}
$$

Calculate the Tasks Execution Order Distances. If two tasks are executed in a different order from the ideal order, a task execution order inversion (EOI) happens. Theory 2 is proposed to judge whether an EOI occurs.

Theory 2. Given a set of tasks $\mathcal{T}$, its ideal execution order is $\widehat{T O I}_{\mathcal{T}}$, and its actual execution order is $\widehat{T O A}_{\mathcal{T}} . \forall \tau_{i}, \tau_{j} \in \mathcal{T}$, a task execution order inversion (EOI) happens only if the following requirements are met:

(1) $\tau_{i} \in \tau_{x_{1}}^{\mathcal{I}^{\prime}} \wedge \tau_{x_{1}}^{\mathcal{I}^{\prime}} \in \widehat{T O I}_{\mathcal{T}}, \tau_{i} \in \tau_{x_{2}}^{\mathcal{A}^{\prime}} \wedge \tau_{x_{2}}^{\mathcal{A}^{\prime}} \in \widehat{T O I}_{\mathcal{T}}$;

(2) $\tau_{j} \in \tau_{y_{1}}^{\mathcal{I}^{\prime}} \wedge \tau_{y_{1}}^{\mathcal{I}^{\prime}} \in \widehat{T O I} \mathcal{T}, \tau_{j} \in \tau_{y_{2}}^{\mathcal{A}^{\prime}} \wedge \tau_{y_{2}}^{\mathcal{A}^{\prime}} \in \widehat{T O I}_{\mathcal{T}}$;

(3) $\tau_{x_{1}}^{\mathcal{I}^{\prime}}<\tau_{y_{1}}^{\mathcal{I}^{\prime}} \wedge \tau_{x_{2}}^{\mathcal{I}^{\prime}}>\tau_{y_{2}}^{\mathcal{I}^{\prime}} \| \tau_{x_{1}}^{\mathcal{I}^{\prime}}>\tau_{y_{1}}^{\mathcal{I}^{\prime}} \wedge \tau_{x_{2}}^{\mathcal{I}^{\prime}}<\tau_{y_{2}}^{\mathcal{I}^{\prime}}$.

Fig 10 shows how to calculate the number of EOIs of an actual execution order. In $\widehat{T O A_{T}^{a}}, T_{8}$ and $T_{9}$ are executed parallelly in different cores, therefore, $\tau_{4}^{\mathcal{A}^{\prime}}$ can be reordered as: $\left\{T_{9}, T_{8}\right\}$. Therefore, compared with $\widehat{T O I_{T}}$, $\widehat{T O A_{T}^{a}}$ schedules $T_{9}$ after $T_{5}, T_{6}$, and $T_{7}$. According to Theory 2 , there are a number of 3 EOIs in $\widehat{T O A_{T}^{a}}$. In $\widehat{T O A_{T}^{b}}$, since the tasks in $\tau_{2}^{\mathcal{A}^{\prime}}$ and tasks in $\tau_{3}^{\mathcal{A}^{\prime}}$ are executed parallelly in different cores, therefore, $\tau_{2}^{\mathcal{A}^{\prime}}$ can be reordered 


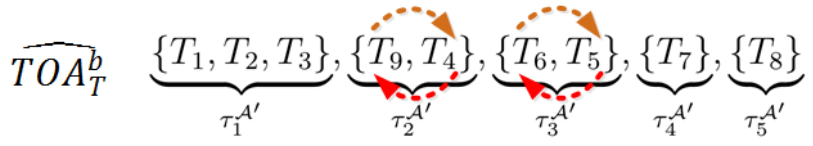

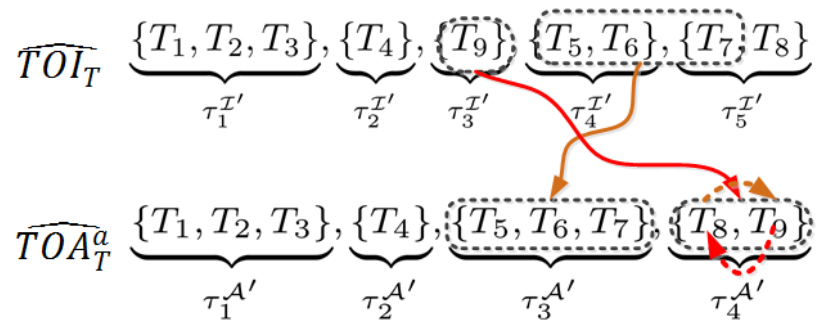

Figure 10: Calculation of the Inversion Number of $\widehat{T O A_{T}^{a}}$ and $\widehat{T O A_{T}^{b}}$

$\left\{T_{4}, T_{9}\right\}$ and $\tau_{3}^{\mathcal{A}^{\prime}}$ can be reordered $\left\{T_{5}, T_{6}\right\}$. Therefore, according to Theory 2. $\widehat{T O A_{T}^{b}}$ has the same order with $\widehat{T O I_{T}}$.

We define the execution order distance from the actual order to the ideal order as the number of execution order inversions of an actual execution order. Therefore, the execution order distance of $\widehat{T O A_{T}^{a}}$ and $\widehat{T O A_{T}^{b}}$ are 3 and 0 , respectively.

Define the Tasks Execution Order Predictability. We use the quotient of the minimum execution order distance over the maximum execution order distance to define the tasks execution order predictability of tasks set $\mathcal{T}$.

Definition 6. Let $V_{O D}$ denote the set of execution order distances of all the actual execution order. If $\exists v_{i} \in V_{O D} \wedge v_{i}=0$, refine $V_{O D}$ as $\widehat{V_{O D}}=$ $\left\{v_{j} \mid v_{j} \in V_{O D} \wedge v_{j} \neq 0\right\}$. If the cardinality of $\widehat{V_{O D}}$ is zero, the predictability of the execution order is 1 , else it is defined as:

$$
\operatorname{Pr}_{\text {TaskOrder }}\left(\widehat{V_{O D}}\right):=\min _{v_{i}, v_{j} \in \widehat{V_{O D}}} \frac{v_{i}}{v_{j}}
$$

The quotient is the quality measure: $\operatorname{Pr}_{\text {TaskOrder }}\left(\widehat{V_{O D}}\right) \in(0,1]$, where 1 means perfectly predictable. In Definition 6 , we refine $V_{O D}$ to $\widehat{V_{O D}}$ to eliminate the order which equals to the ideal order, because in that case, it has no effects on the predictability of task execution. If the execution order is close to 1 , execution order of the task set is highly predictable, and the probability to incur scheduling anomalies is easy to be eliminated as we explained in 
Section 2.2.2. Also, a high predictable execution order allows us to design effective methods to avoid the hazards with mutex, e.g., priority inversion, and deadlock. It should be noted that, a perfectly predictable execution order does not necessarily mean that the actual order is same with the ideal order. However, since the actual execution order is highly predictable in that case, we are able to regulate the actual order by reasonably changing the parameters of tasks (deadlines, periods, and release time, etc.) and scheduling algorithms to achieve predictable execution of the tasks set.

\section{Related Work}

The problem of predictability is firstly discussed in [52], which claims that the predictability should be possible to show, demonstrate, and prove. Henzinger formalizes the notion of predictability as a form of determinis$\mathrm{m}$ [25]. Jensen thinks that something is predictable to the degree that it can be shown in advance [27]. According to Jensen, the predictability is a continuum, having maximum predictability - called determinism - as one end-point. The minimum predictability end-point is that nothing is known at priori about the thing being predicted. Lee argues that predictability is the ability to anticipate the behavior of a system [38, 39, 40, 42]. A property of a model is predictable if there is a terminating procedure that proves that the property is a repeatable property of the model.

The predictability of the discrete dynamical systems is formally defined by measuring the deviation between the actual behavior and the predicted behavior [7]. The time predictability of a system is defined to be related to the difference between best case and lower bound on the one hand and upper bound and worst case on the other hand [53]. The former is called the best case predictability, and the latter is called the worst case predictability. The upper and lower bounds are usually computed off-line through, analysis, simulation, emulation and implementation, that, not during the run-time of the system. The predictability of a task is measured by the best case predictability and the worst case predictability. Kirner and Puschner analyze the possible explanations of the lack of a formal definition of predictability and gives a universal definition as the multiplication of analyzability with stability, including weighting factors [28]. The stability is given by the quotient of BCET over WCET of the time model. For WCET predictability the stability is ignored and the factor for it set to zero. The analyzability, which is now equal to the WCET predictability, as the quotient of WCET over 
WCET bound. Henzinger argues that the programming model should not only be predictable in its function properties (what output does a program compute), but also predictable in its reaction properties (e.g., when does the program provide the output) and in its execution properties (e.g., what resources does the computation consume) [25]. He formalizes the notion of predictability as a form of determinism. An embedded system is time deterministic if, for every timed input stream, the timed output stream that is computed by the system is unique. He also emphasizes that the time determinism refers not only to the input and output values but also to the times at which input values are given to the system and the times at which output values are made available to the environment. No formal and quantifiable definition of predictability or determinism is given in this article.

Grund et al. propose the notion that predictability should capture if, and to what level of precision, a specified property of a system can be predicted by an optimal analysis [21]. They illustrate the proposition through the time predictability, which is defined as the quotient of BCET over WCET with all possible inputs and hardware states. To distinguish hardware- and software-related causes of unpredictability, they introduced the state-induced time predictability and input-induced time predictability by separately considering the sources of uncertainty. They only give the definition of time predictability, and treat the input to be the parameters of the time predictability. However, as we analyzed in Section 2.1, the input predictability is also important in CPS.

In summary, most of the state-of-the-art related researches regard the predictability as a continuum, and only take the predictability of the execution time of the function tasks into consideration. We agree with the idea of regarding the predictability as a continuum. However, we argue that the predictability of CPS should not be limited to the time predictability of function tasks. Both of the I/O behaviors and function task behaviors must be predictable, and both the time property and the order property must be predictable. And the execution time is not suitable to quantify the predictability for the concurrent and preemptive CPS. Therefore, in this paper, we illustrate the reason why the properties we propose must be predictable from different perspectives and define their predictability based on their major sources of uncertainties. 


\section{Summary and Future Work}

CPS have to be predictable to ensure timeliness and improve the reliability of verification and composition. In spite of the great importance of the predictability, there is still no common agreements on the definition of predictability in CPS. The state-of-the-art definitions for predictability, which are studied in the conventional real-time system, are impractical and inadequate for the inherently concurrent and preemptive CPS. Therefore, we intend to propose metrics and definitions of predictability for CPS in this paper. We summarize the predictability requirements of CPS by analyzing characteristics of CPS and the I/O behaviors of typical programming models for CPS, and get the conclusion that both the task execution behavior and the I/O behavior must be predictable. Different from the state-of-the-art predictability definitions, which only consider the time predictability, we argue that the order property also must to be predictable. By analyzing the sources that lead to the uncertainties of the predictable properties, we propose different metrics to measure the predictability of those properties and present formalization of their definitions.

This paper introduces general definitions of predictability for CPS. However, the design and realization of CPS involve different levels of work, such as, design and model, programming and verification, runtime system and hardware support, etc. Therefore, the definitions proposed in this paper need to be optimized for different levels of work in the future. In addition, since our definitions covers new predictable properties, future work should also investigate the relation of them to other properties such as robustness, verification and compositionality.

\section{Acknowledgements}

This work was supported by the National Science Foundation of China under grants (No. 61379040, No. 61272131, No. 61202053), Jiangsu Provincial Natural Science Foundation (No. SBK201240198), Open Project of State Key Laboratory of Computer Architecture, Institute of Computing Technology, Chinese Academy of Sciences (No. CARCH201407). The authors deeply appreciate many reviewers for their insightful comments and suggestions. 


\section{References}

[1] A. Albert. Comparison of Event-Triggered and Time-Triggered Concepts with Regard to Distributed Control Systems. In Embedded World Conf. 2004, pages 235-252, 2004.

[2] S. Andalam, P. S. Roop, and A. Girault. Deterministic, predictable and light-weight multithreading using PRET-C. In Design, Automation \& Test in Europe Conference \& Exhibition (DATE), 2010, pages 16531656, 2010.

[3] P. M. Arenas and H. W. Grote. IEEE 802.11 Throughput and Delay Analysis for mixed real time and normal data traffic. In Ad-Hoc Networking, pages 129-139. Springer, 2006.

[4] P. Axer, R. Ernst, H. Falk, A. Girault, D. Grund, N. Guan, B. Jonsson, P. Marwedel, J. Reineke, C. Rochange, et al. Building timing predictable embedded systems. ACM Transactions on Embedded Computing Systems (TECS), 13(4):82, 2014.

[5] R. Banakar, S. Steinke, B.-S. Lee, M. Balakrishnan, and P. Marwedel. Scratchpad Memory: A Design Alternative for Cache On-chip memory in Embedded Systems. In Proceedings of the 10th International Symposium on Hardware/Software Codesign, pages 73-78. ACM, 2002.

[6] H. Bauer, J.-L. Scharbarg, and C. Fraboul. Worst-case end-to-end delay analysis of an avionics AFDX network. In Proceedings of the Conference on Design, Automation and Test in Europe, pages 1220-1224. European Design and Automation Association, 2010.

[7] N. Bernardes Jr. On the Predictability of Discrete Dynamical Systems. Proceedings of the American Mathematical Society, 130(7):1983-1992, 2002.

[8] G. Berry and G. Gonthier. The ESTEREL synchronous programming language: design, semantics, implementation. Science of Computer Programming, 19(2):87-152, 1992.

[9] J. Berwanger, C. Ebner, A. Schedl, R. Belschner, S. Fluhrer, P. Lohrmann, E. Fuchs, D. Millinger, M. Sprachmann, F. Bogenberger, 
et al. FlexRay-The Communication System for Advanced Automotive Control Systems. SAE Transactions, 110(7):303-314, 2001.

[10] R. J. Bril, J. J. Lukkien, and R. H. Mak. Best-Case Response Times and Jitter Analysis of Real-Time Tasks with Arbitrary Deadlines. In Proceedings of the 21st International conference on Real-Time Networks and Systems, pages 193-202. ACM, 2013.

[11] A. Burns. Preemptive Priority based Scheduling: An Appropriate Engineering Approach. In Advances in Real Time Systems, pages 225-248. S.H. Son, ed. Prentice Hall, 1993.

[12] G. C. Buttazzo. Hard Real-Time Computing Systems: Predictable Scheduling Algorithms and Applications, volume 24. Springer, 2011.

[13] CAN. Controller Area Network CAN, an In-Vehicle Serial Communication Protocol. SAE Press, 20:341-20, 1992.

[14] T. H. Cormen, C. E. Leiserson, R. L. Rivest, and S. Clifford. Introduction to Algorithms. MIT press, 2009.

[15] J. Fraden. Handbook of modern sensors: physics, designs, and applications. Springer Science \& Business Media, 2004.

[16] A. Gamatié. Designing Embedded Systems with the SIGNAL Programming Language: Synchronous, Reactive Specification. Springer Science \& Business Media, 2009.

[17] G. Gershinsky, A. Harpaz, N. Naaman, H. Paz, and K. Shagin. Delay Analysis of Real-Time Data Dissemination. In Proceedings of the 11th communications and networking simulation symposium, pages 205-212. ACM, 2008.

[18] J. B. Goodenough and L. Sha. The Priority Ceiling Protocol: A Method for Minimizing the Blocking of High Priority Ada Tasks, volume 8. ACM, 1988.

[19] R. L. Graham. Bounds on the Performance of Scheduling Algorithms. Computer and Job Scheduling Theory, pages 165-227, 1976. 
[20] D. Grund. Towards a Formal Definition of Timing Predictability. In Workshop on Reconciling Performance with Predictability, Grenoble, France, 2009.

[21] D. Grund, J. Reineke, and R. Wilhelm. A Template for Predictability Definitions with Supporting Evidence. In OASIcs-OpenAccess Series in Informatics, 2011.

[22] R. Ha and J. W. Liu. Validating Timing Constraints in Multiprocessor and Distributed Real-Time Systems. In Distributed Computing Systems, 1994., Proceedings of the 14th International Conference on, pages 162171. IEEE, 1994.

[23] N. Halbwachs, P. Caspi, P. Raymond, and D. Pilaud. The synchronous data flow programming language LUSTRE. Proceedings of the IEEE, 79(9):1305-1320, 1991.

[24] H. M. Hashemian. Measurement of Dynamic Temperatures and Pressures in Nuclear Power Plants. PhD thesis, The University of Western Ontario, 2011.

[25] T. A. Henzinger. Two challenges in embedded systems design: predictability and robustness. Philosophical Transactions of the Royal Society A: Mathematical, Physical and Engineering Sciences, 366(1881):3727-3736, 2008.

[26] S. Issa, H. Sturm, and W. Lang. Modeling of the Response Time of Thermal Flow Sensors. Micromachines, 2(4):385-393, 2011.

[27] E. D. Jensen. Wrong Assumptions and Neglected Areas in Real-Time Systems. In 11th IEEE Symposium on Object Oriented Real-Time Distributed Computing (ISORC), page 356. IEEE, 2008.

[28] R. Kirner and P. Puschner. Time-Predictable Computing. In Software Technologies for Embedded and Ubiquitous Systems, pages 23-34. Springer, 2011.

[29] C. M. Kirsch and R. Sengupta. The Evolution of Real-Time Programming. Handbook of Real-Time and Embedded Systems, pages 11.1-11.15, 2006 . 
[30] C. M. Kirsch and A. Sokolova. The Logical Execution Time Paradigm. In Advances in Real-Time Systems, pages 103-120. Springer, 2012.

[31] M. H. Klein and T. Ralya. An analysis of Input/Output Paradigms for Real-Time Systems. Technical report, CMU/SEI90-TR-19, 1990. https://resources.sei.cmu.edu/asset_files/ TechnicalReport/1990_005_001_15869.pdf.

[32] D. E. Knuth and J. L. Szwarcfiter. A Structured Program to Generate All Topological Sorting Arrangements. Information Processing Letters, 2(6):153-157, 1974.

[33] H. Kopetz. Event-Triggered versus Time-Triggered Real-Time Systems. In Operating Systems of the 90s and Beyond, pages 86-101. Springer, 1991.

[34] H. Kopetz. The Rationale for Time-Triggered Ethernet. In Real-Time Systems Symposium, 2008, pages 3-11. IEEE, 2008.

[35] H. Kopetz. Real-time systems: design principles for distributed embedded applications. Springer Science \& Business Media, 2011.

[36] H. Kopetz and G. Grünsteidl. TTP-A Time-Triggered Protocol for Fault-Tolerant Real-Time Systems. In Fault-Tolerant Computing, 1993. FTCS-23. Digest of Papers., The Twenty-Third International Symposium on, pages 524-533. IEEE, 1993.

[37] H. Kopetz and K. Kim. Temporal Uncertainties in Interactions among Real-Time Objects. In Reliable Distributed Systems, 1990. Proceedings., Ninth Symposium on, pages 165-174. IEEE, 1990.

[38] E. A. Lee. Cyber-Physical Systems - Are Computing Foundations Adequate. In NSF Workshop On Cyber-Physical Systems: Research Motivation, Techniques and Roadmap. Austin, TX, 2006.

[39] E. A. Lee. Computing Foundations and Practice for Cyber-Physical Systems: A Preliminary Report. University of California, Berkeley, Tech. Rep. UCB/EECS-2007-72, 2007.

[40] E. A. Lee. Cyber Physical Systems: Design Challenges. In Object Oriented Real-Time Distributed Computing (ISORC), 2008 11th IEEE International Symposium on, pages 363-369. IEEE, 2008. 
[41] E. A. Lee. Computing Needs Time. Communications of the ACM, 52(5):70-79, 2009.

[42] E. A. Lee. Predictability, Repeatability, and Models for Cyber-Physical Systems, October 2010. Invited talk, Workshop on Foundations of Component Based Design (WFCD) at ESWeek 2010, Scottsdale, AZ.

[43] E. A. Lee, S. Neuendorffer, and M. J. Wirthlin. Actor-Oriented Design of Embedded Hardware and Software Systems. Journal of Circuits, Systems, and Computers, 12(03):231-260, 2003.

[44] X. Li. Worst-case delay analysis of real-time switched Ethernet networks with flow local synchronization. $\mathrm{PhD}$ thesis, Université de Toulouse, France, 2013.

[45] I. Liu, J. Reineke, and E. A. Lee. A PRET Architecture Supporting Concurrent Programs with Composable Timing Properties. In Signals, Systems and Computers (ASILOMAR), 2010 Conference Record of the Forty Fourth Asilomar Conference on. IEEE, 2010.

[46] R. M. Metcalfe and D. R. Boggs. Ethernet: Distributed Packet Switching for Local Computer Networks. Communications of the ACM, 19(7):395-404, 1976.

[47] M. P. Rao and K. Shet. A Research in Real Time Scheduling Policy for Embedded System Domain. CLEI Electronic Journal, VOL12, (2), 2009.

[48] O. Redell and M. Sanfridson. Exact Best-Case Response Time Analysis of Fixed Priority Scheduled Tasks. In Real-Time Systems, 2002. Proceedings. 14th Euromicro Conference on, pages 165-172. IEEE, 2002.

[49] A. Saifullah, Y. Xu, C. Lu, and Y. Chen. End-to-End Communication Delay Analysis in Industrial Wireless Networks. Computers, IEEE Transactions on, 64(5):1361-1374, 2015.

[50] L. Sha, R. Rajkumar, and J. P. Lehoczky. Priority Inheritance Protocols: An Approach to Real-Time Synchronization. Computers, IEEE Transactions on, 39(9):1175-1185, 1990. 
[51] C. Sosna, T. Walter, and W. Lang. Response time of thermal flow sensors with air as fluid. Sensors and Actuators A: Physical, 172(1):15$20,2011$.

[52] J. A. Stankovic and K. Ramamritham. What is Predictability for RealTime Systems? Real-Time Systems, 2(4):247-254, 1990.

[53] L. Thiele and R. Wilhelm. Design for Timing Predictability. Real-Time Systems, 28:157-177, 2004.

[54] A. Vrchoticky and P. Puschner. On the Feasibility of Response Time Predictions - An Experimental Evaluation. In PDCS Project (Esprit BRA Project 3092). Second Year Report, 1991.

[55] J. G. Webster and H. Eren. Measurement, Instrumentation, and Sensors Handbook: Spatial, Mechanical, Thermal, and Radiation Measurement, volume 1. CRC press, 2014.

[56] R. Wilhelm, J. Engblom, A. Ermedahl, N. Holsti, S. Thesing, D. Whalley, G. Bernat, C. Ferdinand, R. Heckmann, T. Mitra, et al. The WorstCase Execution-Time Problem - Overview of Methods and Survey of Tools. ACM Transactions on Embedded Computing Systems (TECS), 7(3):36, 2008.

[57] M. Xie and M. Haenggi. Towards an end-to-end delay analysis of wireless multihop networks. Ad Hoc Networks, 7(5):849-861, 2009.

[58] S.-N. Yeung and J. Lehoczky. End-to-end Delay Analysis for Real-Time Networks. In Real-Time Systems Symposium, 2001.(RTSS 2001). Proceedings. 22nd IEEE, pages 299-309. IEEE, 2001. 


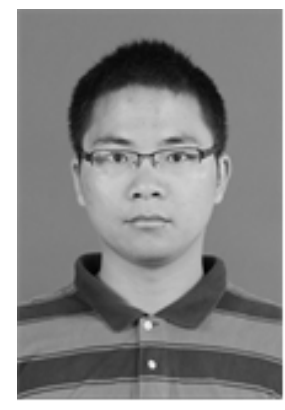

Beilei Sun received the MS degree from University of Science and Technology of China, in 2013. He is currently working toward the PhD degree in the Embedded System Lab of University of Science and Technology of China. His research interests include time-critical systems, DRAM system, and power saving of embedded systems.

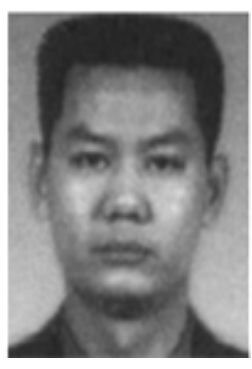

$\mathbf{X i} \mathbf{L i}$ is a professor of Computer Science Department and executive dean of the School of Software Engineering, University of Science and Technology of China. There he directs the research programs in Embedded System Lab, examining various aspects of embedded system with the focus on reliability, performance, availability, flexibility, and energy efficiency. He has led several national key projects of China, several national 863 projects and NSFC projects. He is a member of the IEEE and the ACM, and a senior member of the CCF.

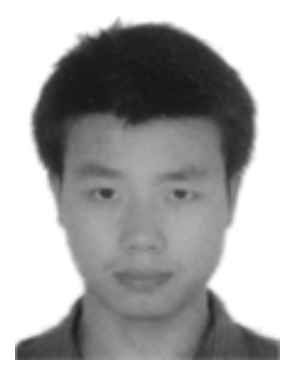

Bo Wan received the BS degree from the HuNan Normal University, in 2013, in software 
engineering. He is a postgraduate in Embedded System Lab of the University of Science and Technology of China. His research interests focus on model of computation and synchronous languages.

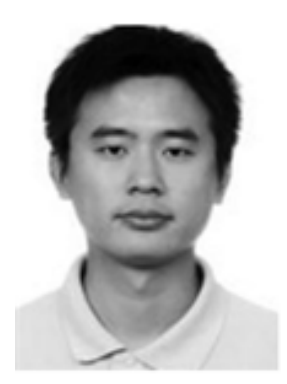

Chao Wang received the BS and PhD degrees in computer science from the University of Science and Technology of China, in 2006 and 2011, respectively. He is a postdoc researcher in Embedded System Lab in the Suzhou Institute of University of Science and Technology of China. His research interests include multicore and reconfigurable computing. He has authored more than 30 publications and patents, including ACM Transactions on Architecture and Code Optimization and FPGA conferences. He is a TPC member of SCC, ICPADS, EUC, ISPA, FPL, ReConfig, and ARC conferences, guest editor for International Journal of Engineering, International Journal of High Performance Systems Architecture, and Journal of Chemical Physics, also a reviewer for DAC, FCCM, FPT, ACM Transactions on Reconfigurable Technology and Systems, ACM Transaction on Embedded Computing, Journal of Systems Architecture, Micropro, and Supercomputing. He is a member of the IEEE, ACM, and CCF.

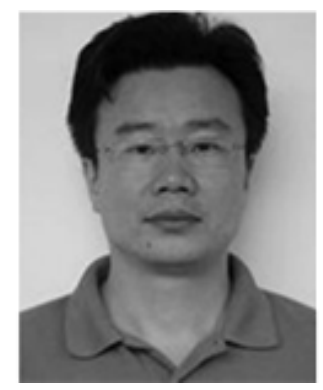

Xuehai Zhou is a professor in the School of Computer Science, University of Science and Technology of China. He is the general secretary of steering committee of Computer College Fundamental Lessons, and technical committee of Open Systems, China Computer Federation. His research interests include various aspects of multicore and distributing systems. He has led 
863 national projects and many NSFC projects. He has published more than 100 international journal and conference articles in the areas of software engineering, operating systems, and distributed computing systems. He is a member of the IEEE and the ACM, and a senior member of the CCF.

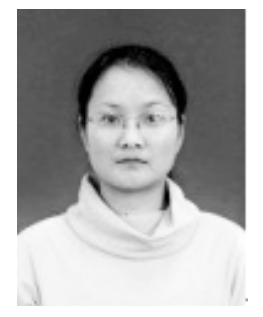

Xianglan Chen is a lecturer in the School of Computer Science, University of Science and Technology of China. She receives her BS and PhD degrees in computer science from the University of Science and Technology of China, in 2000 and 2005, respectively. Her research interests include Operating System and Distributed System. 